Preprints of the

Max Planck Institute for

Research on Collective Goods

Bonn 2008/6

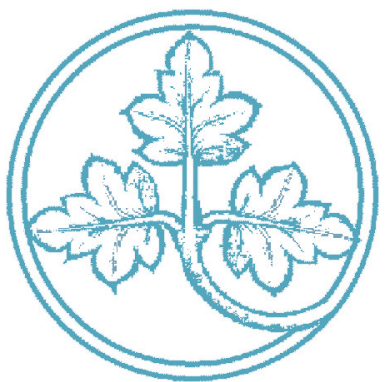

Price Setting in a

Decentralized Market and the Competitive Outcome

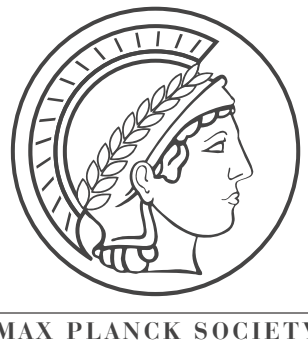




\section{Price Setting in a Decentralized Market and the Competitive Outcome}

Stephan Lauermann

January 2008

Max Planck Institute for Research on Collective Goods, Kurt-Schumacher-Str. 10, D-53113 Bonn http://www.coll.mpg.de 


\title{
Price Setting in a Decentralized Market and the Competitive Outcome
}

\author{
Stephan Lauermann*
}

January 21, 2008

\begin{abstract}
This paper studies a decentralized, dynamic matching and bargaining market: buyers and sellers are matched into pairs. Traders exit the market at a constant rate, inducing search costs (frictions). All price offers are made by sellers. Despite the fact that sellers have all the bargaining power we show that they set competitive prices in the limit when frictions become small. Previous literature has restricted the sellers' bargaining power. We dispense with this restriction and show that the convergence result does not depend on the distribution of bargaining power. Our model allows us to isolate basic market clearing forces that ensure the competitive outcome in the frictionless limit.

For the particular case of homogeneous sellers we characterize the equilibrium price by the familiar Lerner formula. We use this formula to provide comparative static results of the decentralized trading outcome with respect to the level of the search frictions.
\end{abstract}

JEL Classifications: $\quad$ C78, D41, D82, D83

Keywords: Dynamic Matching and Bargaining Games, Decentralized Markets, Non-cooperative Foundations of Competitive Equilibrium, Search Frictions, Rationing.

\footnotetext{
*University of Michigan, Department of Economics, slauerma@umich.edu. I would like to thank Georg Noldeke, Tilman Borgers, Asher Wolinsky, Tymofiy Mylovanov, Martin Hellwig, Philipp Kircher, Mark Satterthwaite for discussions, encouragement, and helpful critique. Financial support by the Deutsche Forschungs Gemeinschaftschaft through SFB/TR 15 and through the GRK 629 is gratefully acknowledged.
} 


\section{Introduction}

It is a common claim that decentralized markets clear and become efficient as frictions vanish. Decentralized markets include the markets for housing, used cars, and labor. Economists often refer to the following informal story as an explanation: Suppose prices in a market are constantly too high. Then some sellers must be rationed and trade less than they desire. This gives them an incentive to decrease their price in order to increase the trading volume, since doing so makes the offer acceptable to more buyers. This incentive makes it impossible to have an equilibrium in which prices are too high to clear the market.

The story relies only on two components: the rationing of sellers and the elasticity of demand. The purpose of the present paper is to isolate these two factors behind the convergence results. We want to differentiate them from additional factors that can be favorable to a competitive outcome. In particular, in the existing literature, the bargaining power of sellers is limited by either allowing buyers to make price offers themselves or by letting several sellers compete against each other (see the discussion of the literature in Section 5.2). By dispensing with these additional assumptions, we provide a more powerful limit result. In addition, we can single out basic market clearing forces as the main factors of the convergence result.

This study uses a dynamic matching and bargaining game that is similar to the model by Gale (1987): There is an infinite number of periods, and in each period there is a large pool of traders who want to trade an indivisible good. The pool consists of a continuum of buyers and sellers: Sellers have costs $c \in[0,1]$, and buyers have valuations $v \in[0,1]$. These types are private information. At the beginning of every period, all sellers and all buyers from the pool are matched into pairs. In each pair, the seller makes a price offer to the buyer. If the buyer accepts the price, they trade and the pair exits the market. If the buyer declines, the match is broken and both traders return to the pool and wait to be rematched with new partners in the next period. While waiting, traders exit with a constant hazard rate $\delta$. The hazard rate introduces costs of waiting for better offers, and we say that $\delta$ is the friction in the market. At the end of every period, an equal mass of new buyers and new sellers enters the market. This inflow of new traders is constant over all periods.

Let $p^{w}$ be defined as the price at which the mass of entering sellers with costs below $p^{w}$ is equal to the mass of entering buyers with valuations above $p^{w}$. This price is the 
competitive or the market clearing price relative to the inflow. The trading outcome is Walrasian if all buyers with valuations above $p^{w}$ and all sellers with costs below $p^{w}$ are able to trade. Our main result characterizes the trading outcome with small $\delta$ : With $\delta \rightarrow 0$, all trades happen at the price $p^{w}$ (Theorem 5 ), and the trading outcome becomes the Walrasian outcome (Corollary 6$){ }^{1}$

We start by considering the case where sellers are homogeneous and their costs are zero. Buyers are heterogeneous and their valuations are distributed between zero and one. The market clearing price is zero; at any price $p^{*}$ above zero, there are more sellers than buyers in the inflow who are willing to trade. When showing convergence to the market clearing price, we proceed in two steps. First, if sellers set a price $p^{*}$ above 0 , some sellers will be rationed: the probability of trading at some time during their life is strictly smaller than one. We show that sellers must remain rationed at the noncompetitive price $p^{*}$ in the limit with $\delta$ converging to zero. Second, if the others are offering $p^{*}>0$, a seller who offers any price $p^{\prime}$ below $p^{*}$ becomes certain to trade in the limit. This is because buyers with valuations between $p^{\prime}$ and $p^{*}$ can never trade with the other sellers. Therefore, these types of buyers make up a strictly positive share of the pool of buyers, and in every single period the seller has a strictly positive chance of being matched with such a buyer. When $\delta$ becomes zero, it becomes certain that the seller will be able to trade some time during his lifetime before he is forced to exit the market. Together, the two steps imply that in the limit a marginal decrease of the price $p^{*}$ increases the trading probability discretely to one, i.e., the elasticity of the trading probability at any $p^{*}$ above zero becomes "infinite" when the exit rate vanishes. Therefore, prices set by sellers have to be zero in the limit.

The convergence result is not immediate. Diamond (1971) shows that even with small trading frictions, sellers can have considerable market power: Given any common price $p^{*}$ set by sellers and any level of $\delta$, buyers with valuations $v>p^{*}$ are willing to pay a premium of $\delta\left(v-p^{*}\right)$ to save on waiting costs. This allows all sellers to mark up the price $p^{*}$ and provides incentives for them to increase their prices. With homogeneous buyers this implies that sellers offer monopolistic prices in the unique equilibrium. This is known as the Diamond paradox. In our model, prices are not monopolistic because buyers are heterogeneous ${ }^{2}$. Sellers are rationed at any price $p^{*}>0$ and they have a countervailing

\footnotetext{
${ }^{1}$ We follow Gale (1987) and the subsequent literature, defining the competitive outcome with respect to the flow.

${ }^{2}$ In addition to heterogeneity, it is also important that we assume that valuations are private information. Prices do not become competitive if valuations are observable; see the discussion of Lauermann (2006a) in Section 5.1.
} 
incentive to decrease their price to reach additional buyers with valuations below $p^{*}$. By giving sellers all the bargaining power, we isolate this incentive from the additional incentives in the existing literature that are due to intermediate bargaining power and direct competition between sellers (see the discussion in Section 5.2). To clearly isolate the market forces of demand and supply in a simple and tractable model is the main contribution of this paper.

When considering heterogeneous sellers with costs distributed between zero and one, it is necessary to account for price dispersion, since sellers with different costs might want to set different prices. Price dispersion implies an additional complication: With dispersed prices, sellers can set prices in such a way as to provide buyers with incentives to accept high prices (by setting high prices most of the time) while balancing the distribution of buyers to avoid accumulating low valuation buyers (by setting low prices some of the time). The main part of the proof with heterogeneous sellers consists of showing that sellers have no incentives to set prices in this way. Instead, a version of the law of one price holds with vanishing $\delta$, and price dispersion ceases to exist. Given the law of one price, we show that prices must be competitive by the fact that sellers (buyers) would be rationed at prices that are too high (too low).

This model differs from the existing literature mainly by positing price setting by sellers. Its basic framework is similar to Gale (1987), who introduced the steady-state dynamic matching and bargaining game with heterogeneous agents, pairwise matching, and an exogeneous inflow of agents. Recent models like those of Inderst (2001) and Satterthwaite and Shneyerov (2007a, 2007b) extended this framework to private information. ${ }^{3}$ Following McAfee (1993) and Satterthwaite and Shneyerov (2007b), we introduce an exogeneous exit rate. Section 5 contains a discussion of our assumptions and of the existing literature.

Finally, in Section 5.3, we use our model to discuss the meaning of "demand" in a dynamic market. During our analysis, we employ two concepts of demand at price $p$ : "Static demand" is exogeneous and it is defined as the mass of buyers in the inflow who have a valuation above $p$. "Dynamic demand" is endogeneous and it is defined as the sellers' probability of trading at a price $p$. It depends on the stock of buyers in the market and on their outside option, i.e., their ability of intertemporal substution. We

\footnotetext{
${ }^{3}$ Moreno and Wooders (2001) also analyze convergence with asymmetric information, but in a nonstationary market with one-time inflow and only two types. Butters' (1977) unfinished typescript also includes asymmetric information and price posting.
} 
relate these two concepts to a recent econometric study of demand in a dynamic market by Nevo and Hendel (2006).

This paper begins with a section introducing the model. The model and the proof technique are illustrated by considering the case of homogeneous sellers. We characterize the unique equilibrium in pure strategies by the Lerner pricing formula. The elasticity of demand is endogenously determined, and a simple argument implies that with vanishing $\delta$ sellers set competitive prices. Then we go on to the heterogeneous case and show convergence to the competitive outcome. A detailed discussion of our modeling choices and extensions is provided in Section 5. In particular, we show which additional assumptions the existing literature makes to ensure convergence, and how these assumptions translate into forces towards market clearance.

\section{Model}

There is a continuum of buyers and sellers who interact in a repeated market over an infinite number of periods. Sellers have one unit of an indivisible good, and their costs of trading are $c \in[0,1]$. Buyers want to buy one unit of the good, and their valuation of the good is $v \in[0,1]$. These types are private information. At the beginning of each period, there is some pool of buyers and sellers. The traders from this pool are randomly matched into pairs consisting of one seller and one buyer. Within each pair, the seller announces a price offer $p \in[0,1]$, and the buyer announces whether he accepts or rejects the offer. If the buyer accepts, they trade and the seller receives a payoff of $p-c$, while the buyer receives a payoff of $v-p$. Next, all the buyers and sellers who have traded exit the pool. A share $\delta$ of all those traders who failed to trade also exits. Finally, new players enter the market and the period ends. The next period starts and proceeds according to the same rules.

The inflow of buyers and the inflow of sellers have a mass of one each. The distribution of valuations among buyers in the inflow is exogeneously given by some c.d.f. $G^{B}(\cdot)$ and similarly, the distribution of costs is given by some distribution $G^{S}(\cdot)$. We assume that the density $g^{B}(\cdot)$ is continuous and strictly positive, so $\left(1-G^{B}(v)\right)$ is strictly decreasing. ${ }^{4}$ The functions $G^{S}$ and $\left(1-G^{B}\right)$ can be interpreted as the supply and demand functions for a Walrasian Auctioneer who clears the market in each period separately. Therefore, we call $G^{S}(\cdot)$ the static supply and $1-G^{B}(\cdot)$ the static demand.

\footnotetext{
${ }^{4}$ We do not assume that the distribution of sellers' costs, $G^{S}(\cdot)$, is strictly increasing, since in the next section we want to give an example with homogeneous sellers who have all $\operatorname{costs} c=0$.
} 
The price at which static supply and demand are equal is called the Walrasian price and is denoted by $p^{w}$ :

$$
G^{S}\left(p^{w}\right)=1-G^{B}\left(p^{w}\right)
$$

The market clearing price is uniquely defined since the right-hand side (supply) is weakly increasing and the left-hand side (demand) is strictly decreasing in the price.

A market constellation is a vector $\sigma=\left[p(\cdot), r(\cdot), \Phi^{S}(\cdot), \Phi^{B}(\cdot), M\right]$, which summarizes the endogeneous parameters of the market: $p(c) \in[0,1]$ is the price offered by a seller of type $c ; r(v) \in[0,1]$ is the highest price accepted by a buyer of type $v$ (see below); $\Phi^{S}(\cdot)$ is the cumulative distribution function of costs in the pool of sellers; $\Phi^{B}(\cdot)$ is the distribution function of buyers' valuations, and $M$ is the total mass of buyers in the pool that is equal to the total mass of sellers in a steady-state. ${ }^{5}$ We say that a market constellation $\sigma^{*}$ constitutes an equilibrium if strategies are mutually optimal given the distribution of types and if the distribution of types in the pool is consistent with the trading strategies and the exogeneous inflow.

First we consider the sellers' decision problem. Let us denote by $D(p \mid \sigma, \delta)$ the probability that the buyer in any given pair accepts an offer $p$, given the market constellation $\sigma$ and the exit rate $\delta$. For future reference, we refer to $D(\cdot \mid \sigma, \delta)$ as the dynamic demand, which depends on the endogeneous distribution of buyers' types in the pool and on their strategies. ${ }^{6}$ To simplify the notation, we keep $\delta$ fixed while presenting the model, and from now on we suppress the dependency on $\delta$. Given $D(\cdot \mid \sigma)$, we derive the probability that a seller is able to trade at some time during his lifetime, $q^{S}(p \mid \sigma)$, the so called lifetime trading probability. We can derive $q^{S}(p \mid \sigma)$ recursively from

$$
q^{S}(p \mid \sigma)=D(p \mid \sigma)+(1-D(p \mid \sigma))(1-\delta) q^{S}(p \mid \sigma) .
$$

as

$$
q^{S}(p \mid \sigma) \equiv \frac{D(p \mid \sigma)}{1-(1-D(p \mid \sigma))(1-\delta)} .
$$

Since there is no discounting, the seller does not care about when he conducts a trade but only about whether he is able to trade before he must exit. ${ }^{7}$ Therefore, the expected payoff to a seller when offering a price $p$ is the product of the selling probability and the

\footnotetext{
${ }^{5}$ For the analysis, we assume that all functions under consideration are measurable. With $\Sigma_{M}$ being the set of measurable functions $f:[0,1] \rightarrow[0,1], \sigma$ is an element of $\Sigma \equiv \Sigma_{M}^{4} \times \mathbb{R}$.

${ }^{6}$ Formally, $D(p \mid \sigma) \equiv \int_{\{v \mid p \leq r(v)\}} d \Phi^{B}(v)$; see below.

${ }^{7}$ Including a discount rate would not change results, see the discussion in Section 5.1.
} 
profit at the price $p$,

$$
U^{S}(p, c \mid \sigma) \equiv q^{S}(p \mid \sigma)(p-c)
$$

We require that $p(c) \in \arg \max U^{S}(\cdot, c \mid \sigma)$ for all $c$ in equilibrium.

To derive the optimal search strategy of a buyer, note that his decision problem is equivalent to the problem of optimal sampling without recall from a known and constant distribution of prices. For this problem, it is well known that the optimal solution can be described by a reservation price $r$, such that a price offer $p$ is accepted if and only if $p \leq r$ (see, e.g., McMillan and Rothschild (1994)). The payoff to a buyer of type $v$ with a reservation price $r$ depends on the expected price offer, $E[p \mid p \leq r, \sigma]$, and the probability to trade at some time during his lifetime. ${ }^{8}$ With $S(r \mid \sigma)$ denoting the probability to receive an acceptable offer $p \leq r$ in any single period, we can derive the lifetime trading probability $q^{B}(r \mid \sigma)$ just as we derived $q^{S}(p \mid \sigma)$ :

$$
q^{B}(r \mid \sigma) \equiv \frac{S(r \mid \sigma)}{1-(1-S(r \mid \sigma))(1-\delta)} .
$$

Payoffs for buyers are given by

$$
U^{B}(r, v \mid \sigma) \equiv q^{B}(r \mid \sigma)(v-E[p \mid p \leq r, \sigma]) .
$$

Let $V^{B}(v \mid \sigma) \equiv \max _{r} U^{B}(r, v \mid \sigma)$ be the maximized expected lifetime payoff. At the optimal reservation price $r(v)$ buyers must be indifferent between acceptance and rejection, so $v-r(v)=(1-\delta) V^{B}(v \mid \sigma)$. Rewriting yields

$$
r(v)=v-(1-\delta) V^{B}(v \mid \sigma)
$$

We restrict attention to stationary equilibria in which the pool of traders does not change over time. Suppose the total mass of sellers and the distribution of their costs at the beginning of a period is given by $M$ and $\Phi^{S}(\cdot)$, respectively, and suppose that the trading strategies are $r(\cdot)$ and $p(\cdot)$. Then the mass of sellers at the end of the period is the sum of the entering sellers and the initial sellers who neither traded nor died, i.e., the mass of sellers having costs below $c$ is

$$
G^{S}(c)+(1-\delta) \int_{0}^{c}(1-D(p(\tau) \mid \sigma)) M d \Phi^{S}(\tau) .
$$

\footnotetext{
${ }^{8}$ Let $E[p \mid p \leq r, \sigma]=r$ if the probability of $p \leq r$ is zero.
} 
Now, $M$ is a steady-state mass and $\Phi^{S}(\cdot)$ is a steady-state distribution of sellers if and only if the pool at the end of the period is the same as the pool at the beginning, i.e., if

$$
M \Phi^{S}(c)=G^{S}(c)+(1-\delta) \int_{0}^{c}(1-D(p(\tau) \mid \sigma)) M d \Phi^{S}(\tau) .
$$

for all $c$. Also, for $\Phi^{S}(\cdot)$ to be a c.d.f., it has to be the case that its value at $c=1$ is normalized to one, $\Phi^{S}(1)=1$. For buyers, a steady state requires that the mass at the end of the period is equal to the mass at the beginning:

$$
M \Phi^{B}(v \mid \sigma)=G^{B}(v)+(1-\delta) \int_{0}^{v}(1-S(r(\tau) \mid \sigma)) M d \Phi^{B}(\tau) .
$$

Summing up, we define an equilibrium to be a constellation that satisfies the above conditions:

Definition 1 An equilibrium $\sigma^{*}$ consists of an optimal pair of strategies and a corresponding steady-state pool, i.e., $\sigma^{*}=\left[p(\cdot), r(\cdot), \Phi^{S}(\cdot), \Phi^{B}(\cdot), M\right]$ must be such that

1. $p(c) \in \arg \max U^{S}\left(p, c \mid \sigma^{*}\right)$ for all $c$,

2. $r(v)=v-(1-\delta) V^{B}\left(v \mid \sigma^{*}\right)$ for all $v$,

3. steady-state conditions (6) and (7) hold for all $c$ and $v$, and $\Phi^{B}(1)=\Phi^{S}(1)=1$.

\section{Homogeneous Sellers: Existence and Characterization}

In this section we analyze the case in which all sellers have zero costs so that the static supply function $G^{S}(\cdot)$ is flat. Static demand $\left(1-G^{B}(\cdot)\right)$ is strictly decreasing. In this case, the market clearing price $p^{w}$ is zero. In addition, $\left(1-G^{B}(\cdot)\right)$ is assumed to be continuously differentiable and strictly concave. ${ }^{9}$ We want to prove that the prices set by sellers converge to zero. The proof's two important building blocks are that (a) sellers are rationed at non-market clearing prices and that (b) in the limit, sellers can increase their trading probability strictly by decreasing their price only marginally. In addition to convergence, we show that we can characterize the equilibrium price offer by the Lerner formula. We use this formula to prove the existence of a unique equilibrium price ${ }^{10}$.

\footnotetext{
${ }^{9}$ Concavity allows us to show existence because we can utilize the sufficiency of the first order condition to derive the optimal price. Concavity is not needed for the characterization result.

${ }^{10}$ Note that the equilibrium definition does not allow sellers of the same type to set different prices. We discuss price dispersion at the end of the next section, showing how to extend the characterization proof for heterogeneous sellers to the homogeneous sellers case with mixed strategies (prices).
} 
Theorem 1 If all sellers have zero costs, then

1. there exists an equilibrium $\sigma^{*}$ for all $\delta \in(0,1)$,

2. for given $\delta$, the equilibrium price $p_{\delta}^{*}=p(0)$ is unique,

3. the price $p_{\delta}^{*}$ decreases if $\delta$ decreases,

4. and the price offer in the limit is competitive, $\lim _{\delta \rightarrow 0} p_{\delta}^{*}=0$.

In the remaining section we prove the theorem. Some of the details are relegated to the appendix.

\subsection{Characterization of the Market}

First, we want to characterize the market constellation in which (i) sellers offer some price $p^{*}$, (ii) buyers use optimal reservation prices, and (iii) the pool is in a steady-state. Thus, we are looking for a market constellation $\sigma\left(p^{*}\right)$ such that, conditional on $p^{*}$, the second and the third equilibrium conditions hold. We start by deriving the reservation prices. For this, note that the payoff of buyers with valuations below $p^{*}$ is zero, while the payoff to buyers with valuations above $p^{*}$ is $v-p^{*}$. Therefore, buyers with valuations below $p^{*}$ accept any price $p \leq v$ since they cannot expect to do any better in the future; thus, $r\left(v \mid p^{*}\right)=v$ for all $v \leq p^{*}$. Buyers with valuations above $p^{*}$ are willing to pay a premium $\delta\left(v-p^{*}\right)$ in order to save the waiting costs, thus their reservation price is strictly above $p^{*}, r\left(v \mid p^{*}\right)=p^{*}+\delta\left(v-p^{*}\right)>0$ for all $v>p^{*}$. Together,

$$
r\left(v \mid p^{*}\right) \equiv \min \left\{v, p^{*}+\delta\left(v-p^{*}\right)\right\}
$$

To characterize the steady state pool, note that buyers with valuations below $p^{*}$ can never trade, but instead they stay in the pool until they die. Their mass in the pool is thus equal to the mass of their inflow $G^{B}\left(p^{*}\right)$, plus the mass of those who entered in the previous period and who survived $(1-\delta) G^{B}\left(p^{*}\right)$, plus those surviving from the next to last period $(1-\delta)^{2} G^{B}\left(p^{*}\right)$, and so on. Together, the mass of buyers with valuations below $p^{*}$ must be $G^{B}\left(p^{*}\right) / \delta=(1-\delta)^{0} G^{B}+(1-\delta)^{1} G^{B}+\ldots$. Buyers with valuations above $p^{*}$ stay in the market for just one period and trade immediately. Thus, their mass in the pool is exactly equal to the mass of their inflow, $\left(1-G^{B}\left(p^{*}\right)\right)$. Taken together, the total mass of all buyers is therefore given by $M=G^{B}\left(p^{*}\right) / \delta+\left(1-G^{B}\left(p^{*}\right)\right)$. The share of buyers with valuations below $p^{*}$ is $\Phi^{B}\left(p^{*}\right)=G^{B}\left(p^{*}\right) / \delta M$. Together 


$$
M\left(p^{*}\right) \equiv G^{B}\left(p^{*}\right) \delta^{-1}+\left(1-G^{B}\left(p^{*}\right)\right)
$$

and

$$
\Phi^{B}\left(v \mid p^{*}\right) \equiv \begin{cases}G^{B}(v) /(\delta M) & \text { if } v \leq p^{*} \\ G^{B}\left(p^{*}\right) /(\delta M)+\left[G^{B}(v)-G^{B}\left(p^{*}\right)\right] / M & \text { if } v>p^{*}\end{cases}
$$

The distribution of sellers is trivially given by $\Phi^{S}\left(c \mid p^{*}\right)=1$ for all $c$.

By construction, $\sigma\left(p^{*}\right)$ satisfies equilibrium conditions (2) and (3). If, in addition, $p^{*}$ maximizes the profit of the sellers, we have found an equilibrium. Therefore $\sigma\left(p^{*}\right)$ is an equilibrium constellation if and only if $p^{*} \in \arg \max U^{S}\left(\cdot, 0 \mid \sigma\left(p^{*}\right)\right)$. As we will see, such a price exists for every $\delta$, and this price is unique. However, before we move to this point, will first consider the equilibrium price when $\delta$ becomes small.

\subsection{Convergence}

We show that prices must become zero when $\delta$ vanishes. The main portion of this task is to derive the limiting lifetime trading probabilities at $p^{*}$ and at any $p^{\prime}$ below it. (Trading probabilities away from the limit are not important here and we will derive them only in the next section.)

If all sellers offer $p^{*}$, then their lifetime trading probability $q^{S}$ is simply the mass of entering buyers with valuations above $p^{*}$. The reason for this is as follows: In a steadystate the mass of buyers who trade must be equal to the mass of sellers who trade. ${ }^{11}$ The mass of buyers who trade is $1-G^{B}\left(p^{*}\right)$, because only those buyers with valuations above $p^{*}$ will find an acceptable price offer. The mass of sellers who trade is $q^{S}$ because each seller trades with probability $q^{S}$, and there is a mass one of sellers who enter the market. Thus, we get ${ }^{12}$

$$
q^{S}\left(p^{*} \mid \sigma\left(p^{*}, \delta\right), \delta\right)=1-G^{B}\left(p^{*}\right) \quad \forall p^{*} \in[0,1] .
$$

For any price $p^{\prime}$ strictly below $p^{*}$, the lifetime trading probability $q^{S}$ can be derived from the per period trading probability $D$. We can find a lower bound on the per period

\footnotetext{
${ }^{11}$ We prove this formally in Lemma 2 , page 17.

${ }^{12}$ In the previous paragraphs we defined $\sigma\left(p^{*}\right)$ for a fixed $\delta$. Here, we vary $\delta$, so we now explicitly include it as an argument in the functions.
} 
trading probability, which is also independent of $\delta$ :

$$
D\left(p^{\prime} \mid \sigma\left(p^{*}, \delta\right), \delta\right) \geq G^{B}\left(p^{*}\right)-G^{B}\left(p^{\prime}\right) \quad \forall p^{\prime} \in\left[0, p^{*}\right) .
$$

Two observations allow us to derive the bound: First, all buyers with valuations between $p^{\prime}$ and $p^{*}$ will accept $p^{\prime}$. Therefore, the trading probability $D$ is at least as large as the share of buyers in the pool who have valuations $v \in\left[p^{\prime}, p^{*}\right]$. Second, the share of these buyers in the pool is at least as large as their share in the inflow: if none of the other types trade, the distribution of types in the pool must be simply the distribution of types in the inflow. Otherwise, if some of the other types do trade, then their share must be even higher in the pool than in the inflow. ${ }^{13}$ Given the bound on the per period trading probability, let us derive the limiting trading probability at the price $p^{\prime}, q^{S}\left(p^{\prime}\right)$ : Since static demand is strictly falling, (12) implies that the trading probability $D\left(p^{\prime}\right)$ is strictly positive. So a seller has a strictly positive probability to trade at $p^{\prime}$ in any given period. When the exit rate converges to zero, the seller can sample for an increasing number of periods. Therefore, the lifetime trading probability at $p^{\prime}$ becomes one:

$$
\lim _{\delta \rightarrow 0} q^{S}\left(p^{\prime} \mid \sigma\left(p^{*}, \delta\right), \delta\right)=1 \quad \forall p^{\prime} \in\left[0, p^{*}\right)
$$

Now, we can see why prices must converge to zero: The trading probability at any price $p^{*}$ is independent of $\delta$ and equal to $\left(1-G^{B}\left(p^{*}\right)\right)$. If $p^{*}$ is not market clearing, then the trading probability is below one, see (11). However, the probability of trading at any $p^{\prime}$ below $p^{*}$ converges to one when $\delta$ becomes zero, see (13). Hence, for $p^{\prime}$ close to $p^{*}$ and for $\delta$ small enough, a deviation from $p^{*}$ to $p^{\prime}$ is profitable. The observation that in the limit even the slightest decrease from $p^{*}$ to $p^{\prime}$ increases $q^{S}$ to one is equivalent to saying that the elasticity of $q^{S}$ becomes infinite for all $p^{*}>0$.

Formally, take a vanishing sequence of exit rates $\left\{\delta_{k}\right\}_{k=1}^{\infty}, \delta_{k} \rightarrow 0$. Given $\delta_{k}$, let $p_{k}^{*} \equiv p_{\delta_{k}}^{*}$ be the equilibrium price. Since $p_{k}^{*} \in[0,1]$, there must be some converging subsequence of prices $\left\{p_{k^{\prime}}\right\}$ with a limit $\bar{p} \in[0,1]$. Along this subsequence, profits at the equilibrium price $p_{k^{\prime}}^{*}$ converge to

$$
\lim _{k^{\prime} \rightarrow \infty} U^{S}\left(p_{k^{\prime}}^{*} \mid \sigma\left(p_{k^{\prime}}^{*}, \delta_{k^{\prime}}\right), \delta_{k^{\prime}}\right)=\bar{p}(1-G(\bar{p})) \quad \forall \bar{p} \in[0,1] .
$$

\footnotetext{
${ }^{13}$ Formally, the share of types $v \in\left[p^{\prime}, p^{*}\right]$ in the pool is defined as $\Phi^{B}\left(p^{*}\right)-\Phi^{B}\left(p^{\prime}\right)$. From (10) it follows that this difference is given by $\left(G^{B}\left(p^{*}\right)-G^{B}\left(p^{\prime}\right)\right)(\delta M)^{-1}$, and from (9) it follows that $(\delta M)^{-1} \geq 1$. Altogether, this implies (12).
} 
Let the "deviation price" $p^{\prime}$ be such that $p^{\prime}$ is strictly below $\bar{p}$. From (13), the lifetime trading probability at $p^{\prime}$ converges to one. The limiting payoff at $p^{\prime}$ are therefore

$$
\lim _{k^{\prime} \rightarrow \infty} U^{S}\left(p^{\prime} \mid \sigma\left(p_{k^{\prime}}^{*}, \delta_{k^{\prime}}\right), \delta_{k^{\prime}}\right)=p^{\prime} \quad \forall p^{\prime} \in[0, \bar{p})
$$

Thus, if $\bar{p}$ is not competitive, then for $p^{\prime}$ close to $\bar{p}$ and for $\delta_{k^{\prime}}$ small enough, profits at $p^{\prime}$ are strictly larger than profits at the equilibrium price $p_{k^{\prime}}^{*}$. This is a contradiction to the definition of $p_{k^{\prime}}^{*}$. Hence, the limit $\bar{p}$ of the subsequence must be zero as claimed. Since the subsequence was chosen arbitrarily, the limit price for every convergent subsequence must be zero and thus the limit of the sequence itself must be zero.

\subsection{Remarks on the Intuition}

A change in $\delta$ changes the composition of the pool and the incentives of the traders at the same time. One cannot therefore derive an intuition by keeping either of the two fixed. For example, an intuition that looks only at the change of the pool might go as follows: "If all sellers offer a common price $p^{*}>0$, then, in the limit with $\delta \rightarrow 0$, buyers with valuations below $p^{*}$ will accumulate and the pool will consist almost entirely of these buyers. Therefore, sellers can no longer expect to sell at $p^{*}$, and hence they decrease their price." The problem with this intuition is that at the same rate at which the pool consists of buyers with lower valuations, sellers become more patient. Indeed, these two effects exactly offset each other such that the relevant lifetime trading probability $q^{S}\left(p^{*}\right)$ is independent of $\delta$ : it is $q^{S}\left(p^{*} \mid \delta\right)=1-G^{B}\left(p^{*}\right)$ for all $\delta$, see (11).

The true reason that sellers want to decrease their price with $\delta \rightarrow 0$ is that it becomes increasingly likely that they will find a buyer with a valuation between $p^{\prime}$ and $p^{*}$ - even if $p^{\prime}$ is just slightly below $p^{*}$. This increase in the matching probability stems from the fact that sellers can sample more often from the pool. This increase is not due to a change in the pool by the accumulation of low valuation buyers. Even if the distribution $\Phi^{B}$ of buyers in the pool remained the same as the distribution $G^{B}$ in the inflow, the sellers' trading probability at $p^{\prime}$ would become one; see the derivation of (12). Therefore, intuition derived solely from the change of the pool misses one of the main driving forces for convergence. ${ }^{14}$

\footnotetext{
${ }^{14}$ Of course, rationing does depend on the decreasing share of buyers with valuations above $p^{*}$. Otherwise, if this share remained constant, the lifetime trading probability at any $p^{*}$ would become one as well, and sellers would not want to decrease their prices; see the discussion of "cloning" in Lauermann (2006b).
} 


\subsection{Existence and Characterization of the Equilibrium Price}

We now prove the existence of an equilibrium for a given $\delta$ by constructing it explicitly. This also allows us to characterize the equilibrium price and to show that this price is unique. Recall that $p^{*}$ is an equilibrium price if and only if $p^{*} \in \arg \max U^{S}\left(\cdot, 0 \mid \sigma\left(p^{*}\right)\right)$. To solve this maximization problem, we first narrow down the set of candidate prices. In particular, only prices in the interval between zero and the highest reservation price can be optimal, i.e., the optimal price must be in $\left(0, r\left(1 \mid p^{*}\right)\right)$ : For prices outside this interval, profits are zero, while profits are strictly positive for prices inside it (since at least some buyers accept such a price). The maximization problem is further simplified by the observation that the payoff $U^{S}\left(p, 0 \mid \sigma\left(p^{*}\right)\right)$ is continuously differentiable in $p$ on this interval and strictly concave. We will show continuous differentiability below. Concavity is shown in the appendix, and this property follows from the assumption that static demand $\left(1-G^{B}(\cdot)\right)$ is concave. Together, the two observations imply that the optimal price is characterized by the first order condition, i.e., we have found an equilibrium price if and only if $\left.\frac{\partial}{\partial p} U^{S}\left(p, 0 \mid \sigma\left(p^{*}\right)\right)\right|_{p=p^{*}}=0$ for some $p^{*} \in\left(0, r\left(1 \mid p^{*}\right)\right)$.

Rewriting the first order condition, ${ }^{15}$ yields the well-known Lerner Formula

$$
\frac{p^{*}-(1-\delta) q^{S}\left(p^{*} \mid \sigma\left(p^{*}\right)\right) p^{*}}{p^{*}}=-\frac{1}{\tilde{\varepsilon}\left(p^{*} \mid \sigma\left(p^{*}\right)\right)},
$$

where $\tilde{\varepsilon}\left(\cdot \mid \sigma\left(p^{*}\right)\right)$ is equal to $-\frac{\partial}{\partial p} D\left(\cdot \mid \sigma\left(p^{*}\right)\right) p D\left(\cdot \mid \sigma\left(p^{*}\right)\right)^{-1}$. This is the elasticity of dynamic demand $D\left(\cdot \mid \sigma\left(p^{*}\right)\right)$ which accounts for the possibility of buyers to substitute intertemporally. The term $(1-\delta) q^{S}\left(p^{*} \mid \sigma\left(p^{*}\right)\right) p^{*}$ can be interpreted as the dynamic opportunity costs of selling the good: Not selling today and offering the good at a price $p^{*}$ from tomorrow onwards yields expected profits of $(1-\delta) q^{S}\left(p^{*} \mid \sigma\left(p^{*}\right)\right) p^{*}$. Hence, the right-hand side is the relative mark up of $p^{*}$ over the dynamic opportunity costs.

The Lerner formula (14) can be written in terms of static demand $\left(1-G^{B}(\cdot)\right)$ by solving for the lifetime trading probability $q^{S}\left(\cdot \mid \sigma\left(p^{*}\right)\right)$ and the elasticity $\tilde{\varepsilon}\left(p^{*} \mid \sigma\left(p^{*}\right)\right)$. We already know the probability $q^{S}$ from (11). The dynamic elasticity $\tilde{\varepsilon}$ can also be derived very easily. It is equal to the static elasticity scaled up by $\delta^{-1}$ :

$$
\tilde{\varepsilon}\left(p^{*} \mid \sigma\left(p^{*}\right)\right)=\frac{1}{\delta} \varepsilon\left(p^{*}\right),
$$

where the elasticity of static demand $\left(1-G^{B}(\cdot)\right)$ is $\varepsilon(p)=-\frac{p g^{B}(p)}{\left(1-G^{B}(p)\right)}$. To see why

\footnotetext{
${ }^{15}$ See the appendix for the algebraic manipulations.
} 
this is the case, we need to know the dynamic demand $D$ and its derivative. To derive demand, we define $v\left(p \mid p^{*}\right)$ as the lowest type who accepts the price $p: v\left(p \mid p^{*}\right) \equiv$ $\inf \left\{v, 1 \mid r\left(v \mid p^{*}\right) \geq p\right\}$. Since $r\left(v \mid p^{*}\right)$ is given by (8), this type is

$$
v\left(p \mid p^{*}\right)= \begin{cases}p^{*}+\frac{1}{\delta}\left(p-p^{*}\right) & \text { if } p \in\left[p^{*}, r\left(v \mid p^{*}\right)\right] \\ p & \text { if } p \in\left[0, p^{*}\right)\end{cases}
$$

The set of buyers who accept a price $p$ is the set of buyers with valuations above $v\left(p \mid p^{*}\right)$. Hence, dynamic demand is $D\left(p \mid \sigma\left(p^{*}\right)\right)=1-\Phi^{B}\left(v\left(p \mid p^{*}\right) \mid p^{*}\right)$. We know the distribution of buyers types' from (10), and the derivative of dynamic demand is

$$
\begin{aligned}
\frac{\partial}{\partial p} D\left(p \mid \sigma\left(p^{*}\right)\right) & =-\Phi^{B}\left(v\left(p \mid p^{*}\right) \mid p^{*}\right) v^{\prime}\left(p \mid p^{*}\right) \\
& = \begin{cases}-\frac{g^{B}\left(v\left(p \mid p^{*}\right)\right)}{M} \frac{1}{\delta} & \text { if } p \in\left[p^{*}, r\left(v \mid p^{*}\right)\right] \\
-\frac{g^{B}\left(v\left(p \mid p^{*}\right)\right)}{\delta M} & \text { if } p \in\left[0, p^{*}\right) .\end{cases}
\end{aligned}
$$

From the characterization of $D\left(p \mid \sigma\left(p^{*}\right)\right)$ and its derivative, the formula (15) for the elasticity $\tilde{\varepsilon}\left(p^{*} \mid \sigma\left(p^{*}\right)\right)$ follows. Note also that the derivative of the dynamic demand is continuous and in particular, the dynamic demand has no kink at $p^{*}$. This might be surprising at first but it follows intuitively from the observation that a seller who decreases his price wins buyers with valuations below $p^{*}$ who never trade and who accumulate at a rate proportional to $\delta^{-1}$, while a seller who increases his price looses buyers who would rather wait for the next period and he looses the types of these buyers at a rate $v^{\prime}\left(p \mid p^{*}\right)=\delta^{-1}$. Finally, continuous differentiability of $D$ implies that payoffs $U^{S}$ are continuously differentiable as assumed in the beginning of this section (since $U^{S}=D p(D+\delta-\delta D)^{-1}$; see the definitions of $q^{S}$ and $U^{S},(2)$ and (3)).

Our knowledge of $q^{S}$ and $\tilde{\varepsilon}$ allows us to rewrite the Lerner formula (14) as ${ }^{16}$

$$
p^{*}\left(1+\left(\frac{1}{\delta}-1\right) G^{B}\left(p^{*}\right)\right)=\frac{\left(1-G^{B}\left(p^{*}\right)\right)}{g^{B}\left(p^{*}\right)}
$$

The remaining parts of the theorem now follow: The existence of a solution $p^{*}$ is implied by the intermediate value theorem, since both sides of the equation are continuous. Given $p^{*}$, we can construct an equilibrium constellation $\sigma^{*}=\sigma^{*}\left(p^{*}\right)$ from (8), (9), and (10). This proves the existence. The uniqueness and monotonicity of the solution follows from

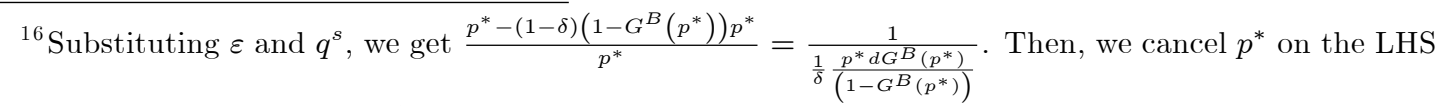
and multiply both sides by $\frac{p^{*}}{\delta}$.
} 
the concavity of the static demand $(1-G)$ : Note that the right-hand side is decreasing in $p^{*}$, since its derivative has the sign of $-g^{2}\left(p^{*}\right)-\left(1-G^{B}\left(p^{*}\right)\right) g^{\prime}\left(p^{*}\right)$, where, by the concavity of $\left(1-G^{B}\right), g^{\prime}$ is strictly positive. The left-hand side is obviously increasing in $p^{*}$. Together, there can be only one $p^{*}$ for which the equality holds. Let this unique price be $p_{\delta}^{*}$. The monotonicity of the terms of the rewritten Lerner formula also implies that the solution $p_{\delta}^{*}$ must be increasing in $\delta$. If $p_{\delta}^{*}$ were decreasing in $\delta$, then our previous discussion of the signs of the change in $p^{*}$ would imply that then the right-hand side would increase in $\delta$, while the left-hand side would decrease in $\delta$ - so the equality would not hold for different $\delta$.

We can use the Lerner Formula to give an alternative proof of convergence. Let $\left\{p_{k^{\prime}}^{*}\right\}$ again be a convergent subsequence of prices with limit $\bar{p}$. Then, if $\bar{p}>0$, the inverse elasticity on the right-hand side of the Lerner formula converges to zero: the dynamic elasticity $\tilde{\varepsilon}$ is equal to the static elasticity $\varepsilon(\bar{p})$ times $\delta^{-1}$. Since the static elasticity $\varepsilon(\bar{p})$ is constant and positive, the inverse dynamic elasticity becomes zero, $\tilde{\varepsilon}_{k^{\prime}}^{-1}=\delta_{k^{\prime}} \varepsilon(\bar{p})^{-1} \rightarrow 0$. The relative mark up on the left-hand side of the Lerner Formula, however, does not converge to zero since the trading probability $q_{k^{\prime}}^{S}(\bar{p})$ is bounded away from one since $\bar{p}$ is not market clearing. Thus, the Lerner formula cannot hold unless $\bar{p}$ is zero. This alternative proof illustrates again how rationing sellers at non-market clearing prices is essential for convergence. However, one needs to first derive the Lerner Formula and this proof is therefore not as direct as the comparison of the lifetime trading probabilities, which we used in our previous proof.

\section{Heterogeneous Sellers}

We now consider heterogeneous sellers. With heterogeneous sellers, we need to account for price dispersion. The main economic contribution of this section is to illustrate the market forces that imply that price dispersion ceases in the limit and that the law of one price holds. At the end of Section 4.2 we show how to use our characterization result with heterogeneous sellers to analyze price dispersion in the case of homogeneous sellers. Technically, an equilibrium can no longer be characterized by a single price but only by a price function. The existence proof is therefore fairly involved and we relegate it to the appendix.

To characterize the equilibria in the limit, we will look at a strictly decreasing sequence of exit rates which converge to zero, $\lim _{k \rightarrow \infty} \delta_{k}=0$. In the first subsection that follows, we will show that for every such $\delta_{k}$ an equilibrium exists. We will also show the 
monotonicity of the equilibrium strategies $p^{*}$ and $r^{*}$. The reader who is not interested in these more technical details might wish to jump directly to the second subsection. There, we select one equilibrium for each $k$, which gives us a sequence of equilibria $\left\{\sigma_{k}^{*}\right\}_{k=1}^{\infty}$. We show that for every such sequence, the support of prices at which trade happens shrinks to a singleton, and hence, a law of one price holds. We then show that this one price must be the Walrasian price $p^{w}$, which is stated in the second theorem. Convergence to the Walrasian Allocation follows as a corollary.

\subsection{Existence and Preliminary Characterization}

In this section, we prepare the analysis of the model with heterogeneous sellers. Now, both, $G^{S}(\cdot)$ and $G^{B}(\cdot)$ are strictly increasing and we assume that they have continuous densities $g^{S}(\cdot)$ and $g^{B}(\cdot)$. We need no longer assume that $\left(1-G^{B}(\cdot)\right)$ is concave. We first prove that prices $p(\cdot)$ and reservation prices $r(\cdot)$ are monotone. Then, we show that for every monotone strategy combination $p(\cdot)$ and $r(\cdot)$ (not just equilibrium strategies), there exists a steady-state pool of traders. We also show that for every monotone strategy combination and for every corresponding steady-state pool, the transfers collectively made by sellers are equal to the transfers received by buyers, and the total mass of sellers who trade is equal to the total mass of buyers who trade. Finally, we prove that an equilibrium exists for all $\delta$. All proofs are collected in the appendix.

The first lemma shows that the reservation prices of buyers are monotone increasing in their valuations, and the prices set by sellers are monotone increasing in costs, if they have costs below the highest accepted price $r^{*}(1)$. Since prices of sellers with costs above $r^{*}(1)$ are never accepted, they have no impact on the equilibrium. Thus, the prices set by these sellers can be changed such that these prices are monotone as well without affecting the equilibrium conditions.

Lemma 1 If $\sigma^{*}=\left[p^{*}, r^{*}, \Phi^{S *}, \Phi^{B *}, M^{*}\right]$ is a steady-state equilibrium then, defining

$$
\tilde{p}(c) \equiv \begin{cases}p^{*}(c) & \forall c \in[0, r(1)) \\ c & \forall c \in\left[r^{*}(1), 1\right],\end{cases}
$$

$\tilde{\sigma}=\left[\tilde{p}, r^{*}, \Phi^{S *}, \Phi^{S *}, M^{*}\right]$ is a steady-state equilibrium, and $\tilde{p}$ and $r^{*}$ are monotone.

Note, if all equilibrium constellations with monotone price functions are competitive, then also all equilibrium constellations in which sellers' prices might be non-monotone 
above $r^{*}(1)$ must be competitive. The restriction to monotone $p$ and $r$, however, makes the analysis a lot easier.

It can also be shown that reservation prices must be strictly increasing with a slope contained between $\delta$ and 1. We define $\Sigma_{r(\cdot)}$ as the set of functions with such a bounded slope, and we define $\Sigma_{p(\cdot)}$ as the set of monotone prices. For every strategy combination of $p$ and $r$ from $\Sigma_{p(\cdot)}$ and $\Sigma_{r(\cdot)}$, we can use a fixed point argument to prove that a pool of traders can be found such that the steady-state conditions hold.

Theorem 2 For every strategy combination $(p(\cdot), r(\cdot)) \in \Sigma_{p(\cdot)} \times \Sigma_{r(\cdot)}$ there is a pool $\Phi^{B}(\cdot), \Phi^{S}(\cdot)$, and $M$ such that the steady-state conditions (6) and (7) hold.

Thus, the steady-state conditions do not restrict the strategy set any further. In models without an exit rate, this is not true, and for some strategies, a steady-state pool fails to exist. In the latter case, the steady-state assumption implies a restriction on the strategies (see the discussion of models with infinitely lived players in Section 5.2).

Intuition suggests that in a steady-state the mass of buyers who trade is equal to the mass of sellers who trade. In addition, the expected payments made by buyers should be equal to the expected payments received by sellers. Indeed, straightforward manipulation of the steady-state conditions shows that this is the case. To state the next lemma, let $\bar{\Sigma}$ be the set of all constellations $\sigma$ such that, given the pricing strategy $p(\cdot)$ and reservation prices $r(\cdot)$, the pool $\Phi^{S}, \Phi^{B}$ and $M$ satisfies the steady-state conditions.

Lemma 2 Mass Balance. Expected payments and the mass of expected trades are equal on both sides of the market, i.e., for all $\sigma \in \bar{\Sigma}$ :

$$
\begin{aligned}
\int_{0}^{1} q^{S}(p(c) \mid \sigma) p(c) d G^{S}(c) & =\int_{0}^{1} q^{B}(r(v) \mid \sigma) E[p \mid p \leq r(v), \sigma] d G^{B}(v) \\
\text { and } \quad \int_{0}^{1} q^{S}(p(c) \mid \sigma) d G^{S}(c) & =\int_{0}^{1} q^{B}(r(v) \mid \sigma) d G^{B}(v) .
\end{aligned}
$$

As discussed in Lauermann (2006b), mass balance does not need to hold in models in which the inflow is not exogeneous as in De Fraja and Sakovics (2001).

Finally, an application of the Kakutani-Fan-Glicksberg Fixed Point Theorem shows an equilibrium exists that for every $\delta$.

Theorem 3 For every $\delta$, there exists an equilibrium constellation $\sigma^{*}$. 


\subsection{The Law of One Price}

To define the support of prices at which trade takes place, let $h_{k}$ be the highest accepted price, $h_{k} \equiv r_{k}(1)$, and let $l_{k}$ be the lowest offered price, $l_{k} \equiv p_{k}(0)$. Now, we state the law of one price.

Theorem 4 For every sequence of steady-state equilibria with $h_{k} \equiv r_{k}(1)$ and $l_{k} \equiv$ $p_{k}(0)$ :

$$
\lim _{k \rightarrow \infty}\left(h_{k}-l_{k}\right)=0 .
$$

In the remainder of this section we prove the theorem by contradiction, using the following line of reasoning: Suppose that, contrary to the theorem, there is some (sub)sequence, indexed by $k^{\prime}$ along which the cutoff prices $h_{k^{\prime}}$ and $l_{k^{\prime}}$ converge to two different limits, i.e., $\lim _{k^{\prime} \rightarrow \infty} h_{k^{\prime}}=h$ and $\lim _{k^{\prime} \rightarrow \infty} l_{k^{\prime}}=l$, with $h>l$. As stated in the introduction, to sustain price dispersion in equilibrium, two opposing conditions must hold: For $r_{k^{\prime}}(1)=h_{k^{\prime}}$ to be optimal, intermediate prices $p \in(l, h)$ must be rare so that the buyer $v=1$ accepts $r_{k^{\prime}}(1)=h_{k^{\prime}}$ instead of waiting for better prices. For $p_{k^{\prime}}(0)=l_{k^{\prime}}$ to be optimal for a seller, intermediate prices must be offered frequently enough: Otherwise, buyers with intermediate valuations would not find trading opportunities and would accumulate in the market. Then, a seller with $c=0$ would want to deviate from the low price $l_{k^{\prime}}$ to some intermediate price. When $\delta_{k^{\prime}}$ is small, the two optimality conditions for the buyers and the sellers cannot both be satisfied, i.e., intermediate prices cannot simultaneously be offered rarely enough for buyers' incentives and frequently enough for sellers' incentives. The following three lemmas formalize this idea, and together they imply the law of one price. The proofs of the lemmas are relegated to the appendix.

During this and subsequent sections, we often refer to the lifetime trading probabilities of types, and we denote these probabilities by capital letters. For this we define $Q^{S}(c \mid \sigma, \delta) \equiv q^{S}(p(c) \mid \sigma, \delta)$ and similarly $Q^{B}(v \mid \sigma, \delta) \equiv q^{B}(r(v) \mid \sigma, \delta) .{ }^{17}$ We abbreviate by using $Q_{k^{\prime}}^{S}(c) \equiv Q^{S}\left(c \mid \sigma_{k^{\prime}}^{*}, \delta_{k^{\prime}}\right)$ and $Q_{k^{\prime}}^{B}(v) \equiv Q_{k^{\prime}}^{B}\left(v \mid \sigma_{k^{\prime}}^{*}, \delta_{k^{\prime}}\right)$.

First, the trading probability at intermediate reservation prices strictly below $h$ must not converge to one. Otherwise, accepting $h$ would not be optimal for a buyer with a valuation of one: By rejecting $h$ and waiting for a lower price instead, he would still trade with a probability of one but at a lower price, which makes him better off. Since buyers with valuations strictly below $h$ can only trade at prices below $h$, this implies that their trading probabilities must not converge to one:

\footnotetext{
${ }^{17}$ Note that we vary the exit rate, and that we therefore include $\delta$ in the arguments.
} 
Lemma 3 Trading probabilities of intermediate types of buyers are bounded away from one:

$$
\lim \sup Q_{k^{\prime}}^{B}(v)<1 \text { for all } v<h
$$

The next two lemmas are the implication of this upper bound on the trading probability. First, payoffs to buyers are bounded. In particular, the limiting payoffs of the intermediate types are strictly smaller than $(v-l)$, since Lemma 3 implies that they cannot become certain to trade at $l$ (or better). Therefore, they accept prices above $l$ in the limit.

Lemma 4 Intermediate types of buyers accept prices above $l$ in the limit:

$$
\liminf r_{k^{\prime}}(v)>l \text { for all } v \in(l, h) .
$$

The second implication of the upper bound on trading probabilities is that intermediate types make up a strictly positive share of the pool in the limit. Intuitively, buyers who are less likely to trade stay in the pool for a longer time and make up a larger share of the pool than those who are more likely to trade:

Lemma 5 Intermediate types of buyers make up a strictly positive share in the limit:

$$
\liminf 1-\Phi_{k^{\prime}}^{B}(v)>0 \text { for all } v \in(l, h) .
$$

Proof of the Theorem: Take any intermediate type $v^{\prime} \in(l, h)$. Suppose a seller having zero costs offers a price equal to the reservation price of this type, $r_{k^{\prime}}\left(v^{\prime}\right)$. All buyers with valuations above $v^{\prime}$ accept these prices. By Lemma 5 , the share of these buyers is strictly positive in the limit. Therefore, the lifetime trading probability of the seller becomes one when $\delta \rightarrow 0$,

$$
\liminf q^{S}\left(r_{k^{\prime}}\left(v^{\prime}\right) \mid \sigma_{k^{\prime}}, \delta_{k^{\prime}}\right)=\liminf \frac{1-\Phi_{k^{\prime}}^{B}\left(v^{\prime}\right)}{1-\left(1-\delta_{k^{\prime}}\right)\left(1-\Phi_{k^{\prime}}^{B}\left(v^{\prime}\right)\right)}=1 .
$$

By Lemma 4 the limiting reservation price of $v^{\prime}$ is larger than $l$. Therefore, the limiting payoff to the zero cost seller who offers $r_{k^{\prime}}\left(v^{\prime}\right)$ will be strictly larger than $l$. If instead the seller would offer the prescribed equilibrium price $p_{k^{\prime}}(0)$, his payoffs would be at most $l$ 
in the limit. So, for $k$ large enough, $p_{k^{\prime}}(0)$ is no longer payoff maximizing and the seller would want to deviate and increase his price to $r_{k^{\prime}}\left(v^{\prime}\right)$ instead. Therefore $\left\{\sigma_{k^{\prime}}\right\}$ cannot be a sequence of equilibrium constellations, yielding the desired contradiction. $Q E D$.

\subsection{Remark on the Intuition for the Law of One Price}

There is an easy but misleading intuition for the law of one price that goes as follows: "There can be no price dispersion in the limit because buyers become more patient with $\delta \rightarrow 0$. Therefore, they would reject all high offers and only accept low prices." This overlooks the fact that the distribution of prices is endogeneous and might change with $\delta$ to keep buyers accepting high offers. Note that our proof is constructed to first show how prices must (and can) be set to actually make buyers accept high prices. Then, the reasoning that such price dispersion is not sustainable is more subtle, and it involves the sellers' incentives to increase prices.

\subsection{Convergence to the Walrasian Price}

Theorem 4 tells us that in the limit, all trades happen at a single price. The next theorem shows that this price is the Walrasian price:

Theorem 5 For every sequence of steady-state equilibria, prices converge to the Walrasian Price:

$$
\lim _{k \rightarrow \infty} p_{k}(c)=p^{w} \forall c<p^{w} \quad \text { and } \quad \lim _{k \rightarrow \infty} r_{k}(v)=p^{w} \forall v>p^{w}
$$

Given the "law of one price," it is sufficient to prove that one of the two boundary prices $l_{k}$ or $h_{k}$ converges to $p^{w}$. Let us prove $\lim _{k \rightarrow \infty} h_{k}=p^{w}$. To do so, we take some convergent subsequence of $\left\{h_{k}\right\}_{k=1}^{\infty}$, indexed by $k^{\prime}$, and call its limit $p^{c}$. First, we show that all sellers with costs below $p^{c}$ must be able to trade in the limit. Second, we show that also all buyers with valuations above $p^{c}$ must be able to trade in the limit. Furthermore, the market clearing price is the only price at which all buyers with valuations above this price and all sellers with costs below this price can trade. Therefore, it must be the case that the limit price $p^{c}$ is equal to $p^{w}$ for every convergent subsequence. And thus, $p^{w}$ must be the limit for the sequence itself.

The first lemma states that the trading probabilities of sellers become one if their costs are below $p^{c}$. The trading probabilities become zero otherwise: 
Lemma 6 For every convergent subsequence $\left\{h_{k^{\prime}}\right\}$ with $\lim _{k^{\prime} \rightarrow \infty} h_{k^{\prime}}=p^{c}$ :

$$
\lim _{k^{\prime} \rightarrow \infty} Q_{k^{\prime}}^{S}(c)=1 \forall c<p^{c} \quad \text { and } \quad \lim _{k^{\prime} \rightarrow \infty} Q_{k^{\prime}}^{S}(c)=0 \forall c>p^{c} .
$$

The proof is quite intuitive: Observe that along the subsequence, for any $p^{\prime}<p^{c}$, buyers with $v \in\left(p^{\prime}, l_{k^{\prime}}\right)$ do not trade, but accumulate instead in the market. ${ }^{18}$ Hence, we know that a seller who offers any price $p^{\prime}$ below $p^{c}$ becomes sure to trade in the limit. Since the equilibrium price offer $p_{k^{\prime}}(c)$ converges to $p^{c}$ by definition, the trading probability at $p_{k^{\prime}}(c)$ must converge to one as well, and we have $Q_{k^{\prime}}^{S}(c) \rightarrow 1$ for all $c<p^{c}$ as claimed. Note that sellers with costs strictly above $p^{c}$ offer only prices $p \geq c>p^{c}$, and even the buyer with the highest reservation price will not accept such prices when $\delta_{k^{\prime}}$ becomes small, since $r_{k^{\prime}}(1) \rightarrow p^{c}$. Thus, sellers with costs above $p^{c}$ will not find a buyer in the limit, and the second part of the lemma follows.

Similarly, we can show that the trading probabilities of buyers with valuations $v>p^{c}$ must converge to one. If not, some buyers would be willing to accept prices strictly above $p^{c}$, contradicting the definition of $h_{k^{\prime}}$ :

Lemma 7 For every convergent subsequence $\left\{h_{k^{\prime}}\right\}$ with $\lim _{k^{\prime} \rightarrow \infty} h_{k^{\prime}}=p^{c}$ :

$$
\lim _{k^{\prime} \rightarrow \infty} Q_{k^{\prime}}^{B}(v)=1 \quad \forall v>p^{c} \quad \text { and } \quad \lim _{k^{\prime} \rightarrow \infty} Q_{k^{\prime}}^{B}(v)=0 \quad \forall v<p^{c}
$$

Proof of Theorem 5: Lemma 6 and 7 imply that in the limit the trading probabilities of sellers and buyers are given by the step functions $1_{c \leq p^{w}}$ and $1_{v \geq p^{v}}$. So we know the mass of players who will trade with $\delta_{k^{\prime}} \rightarrow 0$ :

$$
\begin{aligned}
& \lim _{k^{\prime} \rightarrow \infty} \int_{0}^{1} Q_{k^{\prime}}^{S}(c) d G^{S}(c)=\int_{0}^{1} 1_{c \leq p^{w}} d G^{S}(c)=G^{S}\left(p^{c}\right) \\
& \lim _{k^{\prime} \rightarrow \infty} \int_{0}^{1} Q_{k^{\prime}}^{B}(v) d G^{B}(v)=\int_{0}^{1} 1_{v \geq p^{w}} d G^{B}(v)=1-G^{B}\left(p^{c}\right) .
\end{aligned}
$$

As verified in Lemma 2, in every equilibrium $\sigma_{k^{\prime}}^{*}$, the mass of buyers who trade must be equal to the mass of sellers who trade, i.e., $\int_{0}^{1} Q_{k^{\prime}}^{S}(c) d G^{S}(c)=\int_{0}^{1} Q_{k^{\prime}}^{B}(v) d G^{B}(v)$ for all $k^{\prime}$, and thus,

$$
\lim _{k^{\prime} \rightarrow \infty} \int_{0}^{1} Q_{k^{\prime}}^{S}(c) d G^{S}(c)=\lim _{k^{\prime} \rightarrow \infty} \int_{0}^{1} Q_{k^{\prime}}^{B}(v) d G^{B}(v),
$$

\footnotetext{
${ }^{18}$ This and the following statements are trivial if $p^{c}=0$.
} 
which implies $G^{S}\left(p^{c}\right)=1-G^{B}\left(p^{c}\right)$ at $p^{c}$. The unique price which satisfies this equality is the market clearing price $p^{w}$, and hence, all subsequences $h_{k^{\prime}}$ and $l_{k^{\prime}}$ converge to $p^{w}$. Since all subsequences converge to the same point, the sequence of prices $h_{k}$ itself converges $p^{w} \quad Q E D$.

An immediate corollary of Lemma 6 and 7 is that in the limit those and only those sellers with costs below $p^{w}$ and buyers with valuations above $p^{w}$ trade:

Corollary 6 For every sequence of steady-state equilibria, the outcomes converge to a Walrasian allocation, i.e.,

$$
\begin{array}{llllll}
\lim _{k \rightarrow \infty} Q_{k}^{S}(c)=1 \quad \forall c<p^{w} & \text { and } \quad \lim _{k \rightarrow \infty} Q_{k}^{S}(c)=0 & \forall c>p^{w} \\
\lim _{k \rightarrow \infty} Q_{k}^{B}(v)=1 \quad \forall v>p^{w} & \text { and } \quad \lim _{k \rightarrow \infty} Q_{k}^{B}(v)=0 & \forall v<p^{w}
\end{array}
$$

Proof: Rewrite the Lemmas, substituting $p^{c}=p^{w} \quad Q E D$.

\subsection{The Law of One Price for Homogeneous Sellers}

In the section with homogeneous sellers, we only analyzed equilibria in which all sellers offer the same price $p^{*}$. What about equilibria in which price offers are distributed over some range? One way to answer this question and to accommodate the possibility of price dispersion with homogeneous sellers is to suppose that sellers use distributional strategies which depend on the realization of some random variable. Suppose every seller is characterized by some $i \in[0,1]$ that is drawn at the time of his entry from a uniform distribution, and suppose a seller who draws $i$ offers $p(i)$. With this set-up, we can conveniently analyze equilibria in mixed strategies. In particular, we can use our results about heterogeneous sellers and show that $p(i)$ converges to $p^{w}=0$ for all $i \in[0,1]$ if $\delta \rightarrow 0$. To accomplish this, the proof with heterogeneous sellers could be used almost verbatim. We would first show the law of one price, i.e., the lowest price in the support of price offers converges to the highest accepted price. Here, we could simply repeat the earlier proof. Then, we would show that $l_{k}$ and $h_{k}$ converge to zero. Here, we would need to make small adjustments in the notation since there are no sellers with $\operatorname{costs} c<p^{w}=0$.

\section{Discussion}

We discuss first the setup and the robustness of our result. Then we look at the relation to the existing literature. Finally, we discuss the interpretation of demand in a dynamic 
context.

\subsection{Discussion of the Setup}

Matching Technology. We assume that all entering buyers and sellers are matched into pairs, and that no trader remains unmatched. This assumption allows for the derivation of the lifetime trading probabilities in a very convenient way. Recall that with homogeneous sellers and a common price offer $p^{*}$, the lifetime trading probability was equal to the mass of buyers with valuations above $p^{*}, q^{S}=1-G^{B}\left(p^{*}\right)$; see (11). This simple formulation allows us to directly tell the story of rationing, by connecting the sellers' trading probability with the static demand function and by observing that the trading probability is less than one whenever the price $p^{*}$ is not market clearing. Alternative matching technologies would make the solution of the model less instructive, but they would not change the qualitative results. All that we need for our characterization in the limit is that whenever a set of buyers makes up a strictly positive share of the total pool then the probability that a seller is matched with these buyers is positive.

No Discounting. The exit rate $\delta$ acts like a discount rate by introducing search costs, and in particular, by making these search costs depend on the type: High valuation buyers have more to loose and are more eager to trade. Qualitative results would not change by adding pure time discounting that is proportional to the exit rate, as, e.g., in Satterthwaite and Shneyerov (2007b). However, discounting would drive a wedge between the lifetime trading probability and the discounted lifetime trading probability. While the former can be written in the simple way discussed before, the latter discounted trading probability would be more cumbersome to derive, and would again make the model less instructive.

Price Offers. We restrict sellers to offer simple take-it-or-leave-it prices. In principle, there might be more elaborate selling mechanisms that could raise the seller's payoffs. This is not the case in our quasilinear setup; see the work by Yilankaya (1997), and in particular, the recent work by Mylovanov and Tröger (2007) for continuous type spaces.

Exit Rate. When modelling the evolution of the pool of traders, we follow McAfee (1993), and in particular Satterthwaite and Shneyerov (2007b) and assume that there is some exogeneous exit rate. The main alternative would be to assume that traders literally live infinitely long like in Gale (1987). The assumption that agents live infinitely 
long, however, restricts the set of possible equilibria because it introduces a zero profit condition for sellers. This zero profit condition is not a limit property but it holds even away from the limit and for all levels of frictions. Also, it is independent of any further strategic considerations. Thus, models with infinitely lived agents do not necessarily include the idea that frictions allow traders to enjoy market power in a decentralized market, and that therefore trading is inefficient unless the market becomes frictionless.

To see why the zero profit condition holds let us look at the example from Section 3 where sellers are homogeneous and the market clearing price is zero. To start, note that agents who life infinitely long can leave the market only through trading. Agents who do never trade accumulate in the market and have a mass of infinity. Therefore, one needs to include an entry decision to ensure the existence of a steady-state with finite masses. Second, and again to ensure a steady-state, the mass of entering buyers and sellers must be exactly identical and all traders who decide to enter the market must trade at some point. Now there are two possible equilibrium scenarios: In the first case, all buyers enter, including those with zero valuation. Because even these buyers must be able to trade, sellers must offer prices close to zero. Since sellers would not do so otherwise, this requires that sellers earn zero profits in the first case. In the second case, instead of all buyers, only a mass strictly smaller than one enters. Then, to equalize the mass of entering sellers and buyers, some of the sellers must also choose to stay out of the market. However, sellers will stay out of the market only if they earn zero profits. Therefore, for both cases the zero profit condition holds at all levels of the friction.

No Entry Stage. With infinitely living agents, an entry decision is necessary for technical reasons. We can dispense with it here. If, however, we were to include such an entry stage in a modified model, we could sustain multiple equilibria. For example, in a model with entry, there will typically be a trivial equilibrium in which no trader enters and no trade takes place. Such an equilibrium, however, might be considered unstable because it relies on the assumption that it is impossible for sellers to reach inactive buyers who chose not to enter and who accumulate outside of the pool. If sellers would, for example, be allowed to advertise their prices at some cost per ad to buyers outside the pool and if we let this cost converge to zero, the convergence result could be restored.

Asymmetric Information. Intuition suggests that asymmetric information makes the convergence result harder to attain, because bilateral bargaining between the seller and the buyer must be inefficient if their costs and valuations are private information. This, however, is not the case. In Lauermann (2006a), the present model is altered by 
enabling sellers to observe $v$ before making a price offer. Therefore, bilateral bargaining between the seller and the buyer is efficient. The setup is the same in all other respects. But, without asymmetric information, the convergence results are reversed: prices increase if the friction $\delta$ becomes smaller and the prices set in the limit are not competitive. The reason for this is that price discrimination allows all types of sellers to make strictly positive profits even in the limit. Therefore, marginal sellers with costs close to the market clearing price would not want to trade with marginal buyers, since trading with these buyers would yield only zero profits to them. ${ }^{19}$ The negative result in Lauermann (2006a) not only illustrates the pro-competitive effect of asymmetric information, but it also shows that the convergence result obtained here is not immediately determined from the set-up. For example, low valuation buyers "flood" the market in both variants of the model. Therefore, accumulation of these types cannot be crucial for the results.

Extensions: Non-Steady States and Multiple Goods. It is possible to extend the model in several directions. In particular, one could assume that entry happens only once, in the first period, and that there is no subsequent entry as in Moreno and Wooders (2002). ${ }^{20}$ This would make the pool non-stationary. Similarly, one could extend the model to allow traders to supply and demand several goods as in Gale (2000) or in Atakan (2007). For both extensions, the crucial part of the proof would be to show the "law of one price." Again, the main part would be to show that price dispersion cannot be supported in equilibrium because intermediate types of buyers must not trade with certainty and hence, make up a strictly positive share of the pool. Therefore, sellers would rather deviate and trade with these intermediate types. Having shown the law of one price, one can prove that the price (-vector) must be Walrasian because rationing occurs at all other prices.

\subsection{Existing Literature and Other Market Clearing Forces}

We have argued that rationing and the incentives to reach out for additional buyers are the essential ingredients that guarantee an efficient outcome in the limit. In the existing literature, however, assumptions can be found that give sellers additional incentives to decrease their prices. In the main strand of the literature, ${ }^{21}$ within each pair, both sides

\footnotetext{
${ }^{19}$ That sellers can price discriminate among buyers even in the limit is known as the Diamond Paradox. But price discrimination by itself does not constitute an inefficiency.

${ }^{20}$ Moreno and Wooders assume that both, buyers and sellers, have a chance to make an offer, and they restrict attention to a set of two types.

${ }^{21}$ Mortensen (1982), Rubinstein and Wolinsky (1985) and Gale $(1986,1987)$ initiated the analysis for complete information. A more recent contribution is Mortensen and Wright (2002). Shneyerov and Wong
} 
of the market have a chance to make an offer. In recent models, only sellers can make the offer, but buyers have the chance to simultaneously receive several offers from competing sellers. ${ }^{22}$

For illustration, take a model with homogeneous sellers, where the market clearing price is zero. Suppose that sellers set a common price $p^{*}>0$, even for small $\delta$. As we know, not all sellers will be able to trade at this price and their lifetime trading probability is bounded away from one. This implies furthermore that their profits are strictly smaller than $p^{*}$. Now consider a model with a positive chance that a seller competes directly against the offer of another seller. In this case, there is additional pressure on prices: given the common price level $p^{*}$, any incremental decrease of the price increases the trading probability strictly by undercutting the rivals' prices. Because expected future profits are strictly below $p^{*}$, this increase of the trading probability is profitable. Similarly, consider a model in which buyers can make offers with some probability themselves. Note that, in order to avoid rationing, a seller will accept a low price offer $p^{\prime}$ from a buyer even if it is considerably less than $p^{*}$. Therefore, buyers have the possibility to trade at that price $p^{\prime}$ in the future when it is their turn to make an offer. Moreover, if $\delta$ is close enough to zero, buyers can almost certainly do so. This outside option of trading at a much lower price in the future makes them unwilling to accept an offer $p^{*}$ from the seller. Therefore, sellers are forced to decrease their price offer in order to make it acceptable.

We can distinguish three forces that push prices towards the competitive level: the incentive to reach out to additional buyers analyzed here, the incentive to undercut the competitors, and the outside option for buyers if they have some bargaining power. Rationing on the sellers' side is the common starting point. However, there is an important qualitative difference between the three forces: While the existence of additional buyers at lower prices is a basic feature implied by nothing more than falling demand, the possibility of directly competing offers or the distribution of bargaining power between traders depends on the fine details of the situation and of the model. By showing to what extent the convergence result is independent on these latter details, we provide evidence for the robustness of the prediction that decentralized trading is efficient.

(2007), Serrano (2002), Moreno and Wooders (2002), and Inderst (2001) extended it towards incomplete information.

${ }^{22}$ See Satterthwaite and Shneyerov $(2007 \mathrm{a}, 2007 \mathrm{~b})$ and the literature on noisy search, e.g., Burdett and Judd (1983). 


\subsection{Demand and Supply in a Dynamic Market}

What is "demand" in a dynamic market? There are at least three empirically appealing notions of demand at price $p$ : (i) The mass of buyers in the market who are present today and who place a value above $p$ on the good, (ii) the mass of buyers in the market who are present today and who would actually buy the good at a price $p$, and (iii) the mass of buyers who buy the good at price $p$ if this is the only price of the good in all past and future times. In our model, these notions correspond to (i) the distribution of types in the pool $\left(1-\Phi^{B}(p)\right)$, (ii) dynamic demand $D^{B}(p)$, and (iii) static demand $1-G^{B}(p)$. "Demand" is less of a problem in a static market: If all buyers enter only today and if also trade takes place only today, then the three concepts do not differ.

In a dynamic market, however, these three notions are different as we have seen and one might ask which one is "the right one." Our analysis suggests the following: If a researcher is interested in a market with small frictions, then static supply and demand might be right. We have shown that in the limit, prices are equal to the market clearing price $p^{w}$, which is determined only by static supply and demand. This confirms the view put forward by Gale (1987). ${ }^{23}$ Away from the limit, however, we have seen how both, static and dynamic supply and demand, jointly determine the prices, as illustrated by the Lerner Formula. The distribution of the true willingness to pay, $\Phi^{B}(p)$ by itself does not enter price determination directly. (But indirectly, together with the level of frictions $\delta, \Phi^{B}$ does determine dynamic demand.)

The three notions matter in empirical research. For example, Nevo and Hendel (2006) study demand for laundry detergents. The possibility of storing laundry detergents makes demand more sensitive to temporary price changes than it would be otherwise. Roughly, they estimate dynamic demand by looking at the true revenue at different prices. Then, they estimate static demand, i.e., the level of demand that would emerge if the price would be constantly fixed at some $p$. Since this demand is unobservable, they use structural estimation by identifying the cost of storage and the distribution of types of consumers. This allows them to calculate static demand. Note that similarly in our model knowledge of the distribution of types in the pool, $\Phi^{B}$, and knowledge of the waiting $\operatorname{costs} \delta$, would allow to calculate the trading probability, $q^{S}(p)$, which depends on static demand $\left(1-G^{B}\right)$. Using their estimates, they show that the dynamic elasticity of demand is much higher than the elasticity of static demand. This is consistent with

\footnotetext{
${ }^{23}$ Gale (1987) was the first to point out the problem in defining "demand" in a dynamic context. However, he did not use the concept of dynamic demand and he was concerned mainly with the limiting outcome and whether it is determined through stocks or flows, $\left(\left(1-\Phi^{B}\right)\right.$ or $\left.\left(1-G^{B}\right)\right)$.
} 
our analysis. Recall, that the elasticity of dynamic demand was equal to the elasticity of static demand scaled up by $\delta^{-1}$ (see equation (15)).

Nevo and Hendel point to the relevance of the right choice of "demand": For example, when the Lerner formula is used to estimate the mark-up in an industry, then this estimate is sensitive to whether one chooses the elasticity of dynamic or static demand. The Lerner formula plays a prominent role in merger analysis and therefore the answer to "What is demand?" has important policy implications. Although we look at the extreme case in which many firms compete under capacity constraints, our characterization of pricing via the Lerner formula indicates that oligopolistic pricing decisions might be sensitive to the dynamic elasticity of demand. In general, our model provides a simple tool to discuss the different possible meanings of "demand" in a dynamic market. It could be interesting to extend the model to an oligopolistic market structure.

\subsection{Conclusion}

In our analysis, we proved the asymptotic efficiency of decentralized trading. The basic forces of demand and supply are sufficient to provide incentives for sellers to set market clearing prices when frictions are small. In particular, our intuitive argument appealed to rationing of traders at non-market clearing prices. We have shown that with homogeneous sellers, prices can be characterized by the familiar Lerner formula, i.e., the mark up of prices over costs is proportional to the inverse elasticity of demand. Both the costs and the elasticity are endogeneous: the dynamic costs of trading for sellers include foregone future profits, and the dynamic elasticity of demand includes the possibility of intertemporal substitution. When frictions become small, we see that rationing, together with the increasing elasticity of demand, implies that prices must converge to their competitive level. Finally, we have discussed how to use our model to clarify the meaning of "supply" and "demand" in a dynamic market. 


\section{A Homogeneous Sellers}

We want to show that $U^{S}\left(p, 0 \mid \sigma\left(p^{*}\right)\right)$ is strictly concave on $\left[0, r\left(1 \mid p^{*}\right)\right]$. For this, we characterize the first and second derivative of $q^{S}$ :

$$
\begin{aligned}
\frac{\partial}{\partial p} q^{S}\left(p \mid \sigma\left(p^{*}\right)\right) & =\frac{d(p)[D(p)+\delta-\delta D(p)]-D(p) d(p)[1-\delta]}{[D(p)+\delta-\delta D(p)]^{2}} \\
& =\frac{d(p) \delta}{[D(p)+\delta-\delta D(p)]^{2}},
\end{aligned}
$$

with $d(p)=\frac{\partial}{\partial p} D\left(p \mid \sigma\left(p^{*}\right)\right)$ and

$$
\begin{aligned}
& \frac{\partial^{2}}{\partial p^{2}} q^{S}\left(p \mid \sigma\left(p^{*}\right)\right)= \\
& \frac{d^{\prime}(p) \delta[D(p)+\delta-\delta D(p)]^{2}-\delta 2 d^{2}(p)[D(p)+\delta-\delta D(p)](1-\delta)}{[D(p)+\delta-\delta D(p)]^{2}}
\end{aligned}
$$

with $d^{\prime}(p)=-g^{B \prime}\left(v\left(p \mid \sigma\left(p^{*}\right)\right)\right) \frac{1}{\delta}$ for $p \in\left[p^{*}, r\left(1 \mid p^{*}\right)\right]$ and $d^{\prime}(p)=-g^{B \prime}\left(v\left(p \mid \sigma\left(p^{*}\right)\right)\right)$ for $p \in\left[0, p^{*}\right)$ noting that the derivative of $v\left(p \mid \sigma\left(p^{*}\right)\right)$ is $\frac{1}{\delta}$ and 1 , respectively. The assumption that $\left(1-G^{B}(\cdot)\right)$ is strictly concave implies that $g^{B \prime}(v)>0$ for all $v$. From that, $d^{\prime}<0$, and an inspection of the above equations reveals that the first and second derivative of $q^{S}\left(\cdot \mid \sigma\left(p^{*}\right)\right)$ are strictly negative. Looking at $\frac{\partial^{2}}{\partial p^{2}} U^{S}\left(p, 0 \mid \sigma\left(p^{*}\right)\right)$, which is equal to $\frac{\partial^{2}}{\partial p^{2}} q^{S}\left(p \mid \sigma\left(p^{*}\right)\right) p+2 \frac{\partial}{\partial p} q^{S}\left(p \mid \sigma\left(p^{*}\right)\right)$, this implies that payoffs are strictly concave on the intervals $\left[0, p^{*}\right)$ and $\left.\left(p^{*}, r\left(1 \mid p^{*}\right)\right]\right)$. And by $\frac{\partial}{\partial p} U^{S}\left(p, 0 \mid \sigma\left(p^{*}\right)\right)$ being continuous at $p^{*}$, this implies that profit is strictly concave on the whole interval $\left[0, r\left(1 \mid p^{*}\right)\right]$.

To derive the Lerner formula, note that from $U^{S}\left(p, 0 \mid \sigma\left(p^{*}\right)\right)=\frac{D(p)}{D(p)+\delta-\delta D(p)} p$ we get

$$
\begin{aligned}
\left.\frac{\partial}{\partial p} U^{S}\left(p, 0 \mid \sigma\left(p^{*}\right)\right)\right|_{p=p^{*}} & =\frac{\left(d\left(p^{*}\right) p^{*}+D\left(p^{*}\right)\right)\left(D\left(p^{*}\right)+\delta-\delta D\left(p^{*}\right)\right)-D\left(p^{*}\right) p^{*}(1-\delta) d\left(p^{*}\right)}{\left(D\left(p^{*}\right)+\delta-\delta D\left(p^{*}\right)\right)^{2}} \\
& =\frac{\left(\left(d\left(p^{*}\right) p^{*}+D\left(p^{*}\right)\right)-q^{S}\left(p^{*}\right) p^{*}(1-\delta) d\left(p^{*}\right)\right)}{\left(D\left(p^{*}\right)+\delta-\delta D\left(p^{*}\right)\right)}
\end{aligned}
$$

where we dropped the dependency on $\sigma\left(p^{*}\right)$. Because the denominator of $\frac{\partial}{\partial p} U^{S}\left(p, 0 \mid \sigma\left(p^{*}\right)\right)$ is strictly positive for all $p,\left.\frac{\partial}{\partial p} U^{S}\left(\cdot \mid p^{*}\right)\right|_{p=p^{*}}=0$ if and only if the nominator is zero. 
We can rewrite the nominator as

$$
\begin{gathered}
d\left(p^{*}\right)\left(p^{*}+\frac{\overbrace{\frac{D\left(p^{*}\right)}{d\left(p^{*}\right)}}^{=\frac{\left(1-G^{B}\left(p^{*}\right)\right) M^{-1}}{g^{B}\left(p^{*}(M \delta)^{-1}\right.}}-q^{S}\left(p^{*}\right) p^{*}(1-\delta))}{=} d\left(p^{*}\right) p^{*}[\frac{\left(p^{*}-q^{S}\left(p^{*}\right) p^{*}(1-\delta)\right)}{p^{*}}-\underbrace{\frac{\left(1-G^{B}\left(p^{*}\right)\right) \delta}{p^{*} g^{B}\left(p^{*}\right)}}_{=\tilde{\varepsilon}\left(p^{*} \mid p^{*}\right)^{-1}}],\right.
\end{gathered}
$$

and by $d\left(p^{*}\right)<0$, we get $\left.\frac{\partial}{\partial p} U^{S}\left(\cdot \mid p^{*}\right)\right|_{p=p^{*}}=0$ if and only the Lerner formula (14) holds.

\section{B Heterogeneous sellers}

\section{B.1 Proof of Lemma 1}

First, we prove that we can restrict attention to a subset of $\Sigma$ (the set of measurable constellations; see Footnote 2) when analyzing equilibrium outcomes. We use this to ease notational burden, for the proof of Theorem 2, and to prepare the existence proof by restricting the set of equilibrium candidates. Let $\sigma^{*}=\left[p^{*}, r^{*}, \Phi^{S *}, \Phi^{S *}, M^{*}\right] \in \Sigma$ be an equilibrium. The equilibrium conditions imply restrictions on these functions that we spell out now: We show that $r^{*}(\cdot)$ must have a slope in $[\delta, 1]$, i.e., $r^{*}(\cdot)$ is in the set

$$
\Sigma_{r(\cdot)}=\{f:[0,1] \rightarrow[0,1] \mid f(a)-f(b) \in[\delta(a-b),(a-b)]\} .
$$

For this, note that if the value function is differentiable at some point $\tilde{v}$, then $V^{\prime}\left(\tilde{v} \mid \sigma^{*}\right)=q^{B}\left(r^{*}(\tilde{v}) \mid \sigma^{*}\right)$ by the envelope theorem. Therefore $r\left(\cdot \mid \sigma^{*}\right)=\tilde{v}-(1-\delta) V\left(\tilde{v} \mid \sigma^{*}\right)$ is differentiable at $\tilde{v}$ as well and $r^{\prime}\left(\tilde{v} \mid \sigma^{*}\right)=1-(1-\delta) q^{B}\left(r^{*}(\tilde{v}) \mid \sigma^{*}\right)$; hence, $r^{\prime}\left(\tilde{v} \mid \sigma^{*}\right) \in$ $[\delta, 1]$ at all differentiability points. This restriction on the slope can be generalized to all points by rewriting the optimality condition $V^{B}(a)-V^{B}(b) \geq U^{B}(r(b), a)-$ $U^{B}(r(b), b)$ and its symmetric analogue and by using the definition of $U^{B}(\cdot, \cdot)$; see, e.g., Milgrom and Segal (2002).

Inspecting the steady-state conditions (6) and (7), shows that $M^{*}$ must be in $\left[1, \delta^{-1}\right]$ : 
Rewriting (7) at $v=1$, with $\Phi^{B *}(1)=1$, we get

$$
M^{*}=1+(1-\delta) M^{*} \int\left(1-S\left(r(\tau) \mid \sigma^{*}\right)\right) d \Phi^{B}(\tau)
$$

from where $M^{*} \in\left[1, \delta^{-1}\right]$ follows, substituting $S\left(r(\tau) \mid \sigma^{*}\right) \equiv 1$ to get the lower bound and $S\left(r(\tau) \mid \sigma^{*}\right) \equiv 0$ to get the upper bound. Similarly, one can show that the distribution functions $\Phi^{S}$ and $\Phi^{B}$ are strictly increasing with a bounded slope: Note first, that since the densities $g^{S}$ and $g^{B}$ are strictly positive and continuous by assumption, there are $g_{L}$ and $g_{H}$ such that $g^{B}(v)$ and $g^{S}(c)$ are in $\left[g_{L}, g_{H}\right]$. Rewriting the steady-state condition (7) shows that therefore $\Phi^{B *}(\cdot)$ and $\Phi^{S *}(\cdot)$ are in the set

$$
\Sigma_{\Phi}=\left\{f:[0,1] \rightarrow[0,1] \mid f(a)-f(b) \in\left[g_{L} \delta(a-b), g_{H} \delta^{-1}(a-b)\right]\right\} .
$$

Given monotonicity of reservation prices, we want to show monotonicity of prices $p^{*}(\cdot)$. For this, we use that payoffs satisfy the strict single crossing property. To show that this is true, note that a seller who offers a price $p$ trades with all buyers with a valuation above $v\left(p \mid \sigma^{*}\right) \equiv \inf \left\{v, 1 \mid r^{*}(v) \geq p\right\}$ by monotonicity of $r(\cdot)$. Therefore $D\left(p \mid \sigma^{*}\right)=1-\Phi^{B *}\left(v\left(p \mid \sigma^{*}\right)\right)$. Since $\Phi^{B *}(\cdot)$ and $r^{*}(\cdot)$ are both continuous and strictly increasing, the trading probability $D\left(\cdot \mid \sigma^{*}\right)$ is strictly positive at all prices below the highest reservation price $r(1)$ : for all $p<r(1)$, we have $v\left(p \mid \sigma^{*}\right)<1$ and therefore $D\left(p \mid \sigma^{*}\right)>0$. Hence, for these prices, the lifetime trading probability $q^{S}\left(p \mid \sigma^{*}\right)$ is strictly positive. For all prices above $r^{*}(1)$ trading probabilities are zero: by $v(p)=1$ for all $p \geq r^{*}(1)$ we have $1-\Phi^{B *}(1)=0$. So the relevant range of optimal prices for sellers with costs below $r^{*}(1)$ is $[0, r(1))$. Now we show that profits $U^{S}\left(\cdot, \cdot \mid \sigma^{*}\right)$ satisfy the strict single crossing condition on the domain $\left[0, r^{*}(1)\right)^{2}$, i.e., we show that for all $p_{H}>p_{L}$ and $c_{H}>c_{L}$ with $\left(p_{L}, c_{L}, p_{H}, c_{H}\right) \in\left[0, r^{*}(1)\right)^{4}$ :

$$
U^{S}\left(p_{H}, c_{L} \mid \sigma^{*}\right)-U^{S}\left(p_{L}, c_{L} \mid \sigma^{*}\right) \geq 0 \Rightarrow U^{S}\left(p_{H}, c_{H} \mid \sigma^{*}\right)-U^{S}\left(p_{L}, c_{H} \mid \sigma^{*}\right)>0 .
$$

Rewriting shows that the left-hand side is equivalent to

$$
c_{L}\left(q^{S}\left(p_{L} \mid \sigma^{*}\right)-q^{S}\left(p_{H} \mid \sigma^{*}\right)\right) \geq q^{S}\left(p_{L} \mid \sigma^{*}\right) p_{L}-q^{S}\left(p_{H} \mid \sigma^{*}\right) p_{H} .
$$

Since $q^{S}\left(\cdot \mid \sigma^{*}\right)$ is strictly decreasing in $p$ on $\left[0, r^{*}(1)\right)$ - by $v\left(\cdot \mid \sigma^{*}\right)$ and $\Phi^{B *}(\cdot)$ being strictly increasing - $\left(q^{S}\left(p_{L} \mid \sigma^{*}\right)-q^{S}\left(p_{H} \mid \sigma^{*}\right)\right)>0$. This implies that the left-hand side 
is strictly increasing in costs, and hence

$$
c_{H}\left(q^{S}\left(p_{L} \mid \sigma^{*}\right)-q^{S}\left(p_{H} \mid \sigma^{*}\right)\right)>q^{S}\left(p_{L} \mid \sigma^{*}\right) p_{L}-q^{S}\left(p_{H} \mid \sigma^{*}\right) p_{H},
$$

and this inequality can be rewritten as $U^{S}\left(p_{H}, c_{H} \mid \sigma^{*}\right)-U^{S}\left(p_{L}, c_{H} \mid \sigma^{*}\right)>0$ as claimed. By the monotone selection principle of Milgrom and Shannon (1994), the strict single crossing property implies that all selections from the maximum correspondence $\arg \max _{p} U^{S}\left(p, c \mid \sigma^{*}\right)$ are weakly increasing. Therefore, $p^{*}(\cdot)$ is weakly increasing on $\left[0, r^{*}(1)\right)$. We cannot use optimality conditions to extend the monotonicity of $p^{*}(\cdot)$ to types beyond $r^{*}(1)$ : Every price $p \geq r^{*}(1)$ is optimal for a type $c \geq r^{*}(1)$ since at every such price trading probabilities and profits are zero, while at every price $p<r^{*}(1)$, profits would be strictly negative. Nevertheless, we may simply assume that these types set monotone prices, and without further loss of generality, we may assume that they set prices equal to their costs.

Let $\Sigma_{+}$be the set of weakly increasing functions and define the set $\tilde{\Sigma}$

$$
\tilde{\Sigma} \equiv \Sigma_{+} \times \Sigma_{r(\cdot)} \times \Sigma_{\Phi} \times \Sigma_{\Phi} \times\left[1, \delta^{-1}\right]
$$

We summarize our findings in a lemma. It states that every equilibrium $\sigma^{*}$ is equivalent to an equilibrium $\tilde{\sigma}$, which is in the set $\tilde{\Sigma}$, changing $p^{*}(\cdot)$ to $\tilde{p}(\cdot)$ on $\left[r^{*}(1), 1\right]$, as described before:

Lemma 8 If $\sigma^{*}=\left[p^{*}, r^{*}, \Phi^{S *}, \Phi^{S *}, M^{*}\right]$ is a steady-state equilibrium then, with

$$
\tilde{p}(c) \equiv \begin{cases}p^{*}(c) & \forall c \in[0, r(1)) \\ c & \forall c \in\left[r^{*}(1), 1\right],\end{cases}
$$

$\tilde{\sigma}=\left[\tilde{p}, r^{*}, \Phi^{S *}, \Phi^{S *}, M^{*}\right]$ is a steady-state equilibrium and $\tilde{\sigma} \in \tilde{\Sigma}$.

Lemma 1 follows immediately.

\section{B.2 Proof of Theorem2}

See the remark following the existence proof on page 44 . 


\section{B.3 Proof of Lemma 2}

Take some $\sigma \in \bar{\Sigma}$, i.e., some strategy combination and some corresponding steadystate pool. We show the identity of trading masses by algebraic manipulation, dropping the dependency on $\sigma$ for brevity, with $c(v)=\sup \{c, 0 \mid p(c) \leq r(v)\}$ and $v(c)=$ $\inf \{v, 1 \mid r(v) \geq p(c)\}$ :

$$
\begin{aligned}
\int_{0}^{1} q^{S}(p(c)) d G^{S}(c) & =\int_{0}^{1} \frac{D(p(c))}{D(p(c))+\delta-\delta D(p(c))} d G^{S}(c) \\
& =\int_{0}^{1} M D(p(c)) d \Phi^{S}(c)=\int_{0}^{1} M\left[\int_{v(c)}^{1} d \Phi^{B}(v)\right] d \Phi^{S}(c) \\
& =\int_{0}^{1} M\left[\int_{v(c)}^{1} \frac{d G^{B}(v)}{M(S(r(v))+\delta-\delta S(r(v)))}\right] d \Phi^{S}(c) \\
& =\int_{0}^{1}\left[\int_{0}^{c(v)} d \Phi^{S}(c)\right] \frac{1}{(S(r(v))+\delta-\delta S(r(v)))} d G^{B}(v) \\
& =\int_{0}^{1} S(r(v)) \frac{1}{(S(r(v))+\delta-\delta S(r(v)))} d G^{B}(v)=\int_{0}^{1} q^{B}(r(v)) d G^{B}(v)
\end{aligned}
$$

and similarly the identity of expected payments follows from:

$$
\begin{aligned}
\int_{0}^{1} p(c) q^{S}(p(c)) d G^{S}(c) & =\int_{0}^{1} p(c) M D(p(c)) d \Phi^{S}(c) \\
& =\int_{0}^{1} M\left[\int_{v(c)}^{1} \frac{1}{M(S(r(v))+\delta-\delta S(r(v)))} d G^{B}(v)\right] p(c) d \Phi^{S}(c) \\
& =\int_{0}^{1}\left[\int_{v(c)}^{1} \frac{1}{S(r(v))} d G^{B}(v) q^{B}(r(v))\right] p(c) d \Phi^{S}(c) \\
& =\int_{0}^{1} q^{B}(r(v)) \frac{1}{S(r(v))}\left[\int_{0}^{c(v)} p(c) d \Phi^{S}(c)\right] d G^{B}(v) \\
& =\int_{0}^{1} q^{B}(r(v)) E[p \mid p \leq r(v)] d G^{B}(v)
\end{aligned} \text { QED. }
$$

\section{B.4 Proof of Existence}

Finally, we show that an equilibrium exists for every $\delta$. With heterogeneous sellers, we cannot reduce the existence problem to a one-dimensional fixed point problem as we did for homogeneous seller; we have to prove the existence of a fixed point in the function space $\tilde{\Sigma}$. Instead of the intermediate value theorem, we therefore use the Kakutani-FanGlicksberg theorem. We first introduce some notation: We describe the pool by the 
mass of buyers with valuations above $v, M^{B}(v)$ and the mass of sellers with costs below $c, M^{S}(c)$. The total mass of buyers is $M^{B}(0)$ and the total mass of sellers is $M^{S}(1)$. Throughout the proof we do not require $M^{B}(0)$ to be equal to $M^{S}(1)$. Afterwards we show that if we have found an equilibrium such that the appropriate steady-state conditions hold, these two masses must be equal. With this new notation, the market is characterized by a quartuple of functions $\omega=\left[r(\cdot), p(\cdot), M^{S}(\cdot), M^{B}(\cdot)\right]$. Given $\omega$, we now derive a response operator $K$. This operator consists of the best responses for sellers and buyers, $K^{p}[\omega]$ and $K^{r}[\omega]$, and the pool responses $K^{S}[\omega]$ and $K^{B}[\omega]$. The former will consist of ex ante optimal strategies $p(\cdot)$ and $r(\cdot)$. The latter pool responses are the masses of traders in the pool that will result at the end of a period if the pool at the beginning of the period is described by $M^{S}(\cdot), M^{B}(\cdot)$ and if they trade according to $p(\cdot)$ and $r(\cdot)$. If $\omega^{*}$ is a fixed-point of $K, \omega^{*} \in K\left[\omega^{*}\right]$, traders play mutual best responses and the pool is in a steady-state, i.e., $\omega^{*}$ is an equilibrium. We show that such a fixed point exists and show that $\omega^{*}$ corresponds to an equilibrium $\sigma^{*}$ as originally defined. The main technical challenge is the proof of continuity of the pool-response operators; see Lemma 14.

To prepare for the fixed point theorem, we restrict the set of candidate strategies and distributions under consideration. Observing that $M^{S}(\cdot)$ corresponds to $\Phi^{S}(\cdot) M$, the restrictions on distributions of types become now restrictions on masses, and we define analogously to $\Sigma_{\Phi^{S}}$ and $\Sigma_{\Phi^{B}}$ :

$$
\begin{aligned}
\Sigma_{M^{S}} & \equiv\left\{M^{S}(\cdot):[0,1] \rightarrow\left[0, \delta^{-1}\right] \mid \frac{M^{S}(a)-M^{S}(b)}{a-b} \in\left[g_{l}, g_{h} \delta^{-1}\right], \forall a \neq b\right\} \\
\Sigma_{M^{B}} & \equiv\left\{M^{B}(\cdot):[0,1] \rightarrow\left[0, \delta^{-1}\right] \mid \frac{M^{B}(b)-M^{B}(a)}{a-b} \in\left[g_{l}, g_{h} \delta^{-1}\right], \forall a \neq b\right\},
\end{aligned}
$$

and the domain of $K$ is

$$
\Omega \equiv \Sigma_{p(\cdot)} \times \Sigma_{r(\cdot)} \times \Sigma_{M^{S}} \times \Sigma_{M^{B}}
$$

Because all functions in $\Omega$ are integrable, we use the integral norm $\|f(\cdot)\|_{1}=$ $\int_{0}^{1}|f(t)| d t$ such that $\Omega$ becomes a subspace of $L_{1} \cdot{ }^{24}$

To define payoffs, note that the share of buyers with valuations above $v$ is $M^{B}(v) M^{B}(0)^{-1}$ and we define $D(p \mid \omega) \equiv M^{B}(v(p \mid \omega)) M(0)^{-1}$ and similarly $S(r \mid \omega) \equiv M^{S}(c(r \mid \omega)) M^{S}(1)^{-1}$

\footnotetext{
${ }^{24}$ As usual, we continue working with the function space itself, rather than the corresponding space of equivalence classes. Two functions are equivalent under $\|\cdot\|_{1}$ if they are equal almost everywhere.
} 
with the generalized inverses $c(\cdot \mid \omega)$ and $v(\cdot \mid \omega)$ defined as before: $c(p \mid \omega)=\sup \{c, 0 \mid p(c) \leq p\}$ and $v(p \mid \omega)=\inf \{v, 1 \mid r(v) \geq p\}$. Lifetime trading probabilities for a given $\omega$ are

$$
q^{B}(r \mid \omega) \equiv \frac{S(r \mid \omega)}{1-(1-S(r \mid \omega))(1-\delta)} \text { and } q^{S}(p \mid \omega) \equiv \frac{D(p \mid \omega)}{1-(1-D(p \mid \omega))(1-\delta)} ;
$$

and payoffs are

$$
U^{S}(p, c \mid \omega) \equiv q^{S}(p \mid \omega)(p-c) \text { and } U^{B}(r, v \mid \omega) \equiv q^{B}(r \mid \omega)(v-E[p \mid p \leq r, \omega]) .
$$

Ex ante expected payoffs to sellers are $\Pi(p(\cdot) \mid \omega)$, and interim maximized payoffs to buyers are $V^{B}(v)$ :

$$
\begin{aligned}
\Pi(p(\cdot) \mid \omega) & \equiv \int_{0}^{1} q^{S}(p(c) \mid \omega)(p(c)-c) d G^{S}(c) \\
\text { and } \quad V^{B}(v) & \equiv \max _{r} U^{B}(r, v \mid \omega) .
\end{aligned}
$$

Now we define the operator $K$. The sellers' best response correspondence is defined as

$$
K^{p}[\omega] \equiv \arg \max _{p(\cdot) \in \Sigma_{p(\cdot)}} \Pi(p(\cdot) \mid \omega),
$$

and $K^{p}[\omega] \in \Sigma_{p(\cdot)}$ by definition. With $r(v \mid \omega)=v-(1-\delta) V^{B}(v \mid \omega)$, the buyers' best response is given by

$$
K^{r}[\omega] \equiv r(\cdot) .
$$

Inspection of $V^{B}(v \mid \omega)=q^{B}(r(v) \mid \omega)(v-E[p|p \leq r(v)| \omega])$ shows that $r(\cdot \mid \omega)$ must have a slope between $\delta$ (if $q^{B}(r(v) \mid \omega)=1$ ) and 1 (if $q^{B}(r(v) \mid \omega)=0$ ), i.e., $K^{r}[\omega] \in \Sigma_{r(\cdot)}$.

In analogy to the steady-state conditions, define pool response operators by

$$
\begin{aligned}
K^{S}(c \mid \omega) & \equiv G^{S}(c)+\int_{0}^{c}(1-\delta)(1-D(p(t) \mid \omega)) d M^{S}(t) \\
K^{B}(p \mid \omega) & \equiv\left(1-G^{B}(v)\right)+\int_{v}^{1}(1-\delta)(1-S(r(t) \mid \omega)) d M^{B}(t),
\end{aligned}
$$

where $K^{S}(c \mid \omega)$ is the mass of sellers at the end of the period, consisting of the inflow $G^{S}(c)$ and those sellers who neither trade nor die. Similarly, $K^{B}(v \mid \omega)$ is the mass of buyers at the end of the period, consisting of the new inflow and the remaining buyers from the beginning. To check that $K^{S}$ maps $\Sigma_{M^{S}}$ into itself, note that $K^{S}(1 \mid \omega)$ attains 
its maximal value if no seller trades and then $K^{S}(1 \mid \omega) \leq 1+(1-\delta) M^{S}(1) \leq \delta^{-1}$. The slope of $K^{S}(\cdot \mid \omega)$ is maximal at $d K^{S}(t)=g_{h} \delta^{-1}$ and minimal at $d K^{S}(t)=g_{l}+0$. Therefore, $K^{S}[\omega] \in \Sigma_{M^{S}}$. Reasoning similarly for buyers and adding our observations on the best response operators, we have that $K[\cdot]$ is a self map of $\Omega$ :

$$
K[\omega] \equiv K^{p} \times K^{r} \times K^{S} \times K^{B}: \Omega \rightrightarrows \Omega
$$

We want to prove that $K$ has a fixed point $\omega^{*}$, using the Kakutani-Fan-Glicksberg fixed point theorem. The theorem states that if $\Omega$ is a non-empty, convex, and compact subset of a locally convex Hausdorff space, and if $K$ has a closed graph and nonempty, convex values, then $K$ has a fixed point (see Aliprantis, Border, 1994, p484). In the following lemmas, we prepare the proof by showing that the functions $K^{r}, K^{S}, K^{B}$ are continuous in $\omega$ and that the correspondence $K^{p}$ has convex values and a closed graph.

We use Berge's Maximum Theorem to show that the best response correspondence $K^{p}$ is upper hemicontinuous with compact non-empty values. This implies that $K^{p}$ has a closed graph (see Aliprantis, Border, p. 473 and p. 465). To apply Berge's Theorem, we need to show that expected profits are continuous in $p(\cdot)$ and $\omega$, which will follow from reservation prices being continuous and strictly increasing. Then we show convexity, using the fact that for all $c \in[0, r(1)]$ the best response correspondence is essentially unique (because payoffs satisfy the strict single crossing condition) and for all $c \in(r(1), 1]$, all elements of $K^{p}$ and all their convex combinations yield zero profits:

Lemma $9 K^{p}[\cdot]$ has a closed graph and it is non-empty and convex valued.

Proof: Trading probabilities $q^{S}(p \mid \cdot)$ are continuous for all $p$ in $\omega$, because $q^{S}(p \mid \cdot)$ is a continuous function of $M^{B}(v(p \mid \cdot))$ and $M^{B}(v(p \mid \cdot))$ is continuous in $\omega$, i.e., if $\omega_{N} \rightarrow \bar{\omega}=\left[\bar{p}, \bar{r}, \bar{M}^{S}, \bar{M}^{B}\right]$, then $M_{N}^{B}\left(v\left(p \mid \omega_{n}\right)\right) \rightarrow \bar{M}^{B}(v(p \mid \bar{\omega}))$. Note that $v(p \mid \bar{\omega}) \equiv$ $\inf \{v, 1 \mid \bar{r}(v) \geq p\}$, i.e., $v(p \mid \omega)$ is a mapping from $\Omega$ into $[0,1]$. Continuity follows because $r_{N}(v)$ is strictly increasing with a slope bounded from below and hence, $v\left(\cdot \mid \omega_{N}\right)$ is strictly increasing for all $N$. This implies that $v\left(p \mid \omega_{N}\right) \rightarrow v(p \mid \bar{\omega})$ for all $p$. Likewise, $M_{N}^{B}(\cdot)$ is strictly increasing and hence the composite function $M_{N}^{B}\left(v\left(p \mid \omega_{N}\right)\right)$ converges, as claimed.

By the dominated convergence theorem, continuity of $q^{S}(p \mid \cdot)$ for a given price $p$ implies that expected payoffs $\Pi(p(\cdot) \mid \cdot)$ are continuous in $\omega$ for a given function $p(\cdot)$. 
Similarly, expected payoffs are continuous in one's own price function $p(\cdot)$. Therefore Berge's maximum theorem applies.

Now we want to show that $K^{p}$ has convex values. Let the highest type who can possibly trade with positive payoffs be $\bar{c}=\sup \left\{c \mid q^{S}(c \mid \omega)>0\right\}$. As a first step, we show that all (ex ante) optimal pricing functions are equivalent (a.e. identical) on $[0, \bar{c}]$. Take any $p(\cdot) \in K^{p}[\omega]$. Then $p(c)$ is interim optimal for almost every type, i.e., $p(c) \in$ $\arg \max U^{S}(c, p \mid \omega)$ for almost every $c$, and in particular, at every point of continuity: Suppose not, then for some pair $p^{\prime}$, and $c^{\prime}, U^{S}\left(c^{\prime}, p\left(c^{\prime}\right) \mid \omega\right)<U^{S}\left(c^{\prime}, p^{\prime} \mid \omega\right)$. By continuity of $U^{S}(\cdot, \cdot \mid \omega)$ in $p$ and $c$, and by continuity of $p(\cdot)$ at $c^{\prime}$, there is a neighborhood $B_{\delta}\left(c^{\prime}\right)$ such that $U^{S}(c, p(c) \mid \omega)<U^{S}\left(c, p^{\prime} \mid \omega\right)$ for all $c \in B_{\delta}\left(c^{\prime}\right)$. Because $B_{\delta}\left(c^{\prime}\right)$ has strictly positive mass, this implies that $p(\cdot)$ is not profit maximizing ex ante, a contradiction.

Take two functions $p_{1} \in K^{p}[\omega]$ and $p_{2} \in K^{p}[\omega]$ and some $c^{\prime}<\bar{c}$ such that $p_{1}\left(c^{\prime}\right)$ and $p_{2}\left(c^{\prime}\right)$ are (interim) optimal, i.e., $p_{i}\left(c^{\prime}\right) \in \arg \max U^{S}\left(p, c^{\prime} \mid \omega\right), i \in\{1,2\}$. Suppose that $p_{1}\left(c^{\prime}\right) \neq p_{2}\left(c^{\prime}\right)$ and, without loss of generality, suppose $p_{1}\left(c^{\prime}\right) \equiv p_{1}<p_{2} \equiv p_{2}\left(c^{\prime}\right)$. We show that $c^{\prime}$ must be a jump point for both functions. For all prices $p^{+}$above $p_{1}$, the optimality of $p_{1}$ implies $U^{S}\left(p_{1}, c^{\prime} \mid \omega\right) \geq U^{S}\left(p^{+}, c^{\prime} \mid \omega\right)$. Since payoffs satisfy the strict single crossing property, all types $c^{-}$below $c^{\prime}$ strictly prefer $p_{1}$ to any such $p^{+}$, i.e., $U^{S}\left(p_{1}, c^{-} \mid \omega\right)>U^{S}\left(p^{+}, c^{-} \mid \omega\right)$. Similarly, the optimality of $p_{2}$ for $c^{\prime}$ implies that all types $c^{+} \in\left(c^{\prime}, \bar{c}\right)$ prefer $p_{2}$ strictly to any $p^{-}<p_{2}$. Hence, optimal prices for types to the left of $c^{\prime}$ are below $p_{1}$, and optimal prices to the right are above $p_{2}$. Finally, if some (single) type $c^{-}<c^{\prime}$ plays a suboptimal price $p_{2}\left(c^{-}\right)>p_{1}$, all $c \in\left(c^{-}, c^{\prime}\right)$ must play prices above $p_{1}$ by monotonicity of $p_{2}(\cdot)$. But then a strictly positive mass of types sets a strictly suboptimal price, and $p_{2}(\cdot)$ fails ex ante optimality. Therefore $p_{i}\left(c^{\prime}-0\right) \leq p_{1}<p_{2} \leq p_{i}\left(c^{\prime}+0\right),{ }^{25} i \in\{1,2\}$, and $c^{\prime}$ is a jump point as claimed. To sum up, the two functions can at most be different on the zero measure set of points at which either one of these functions jumps or at which either one of them does not prescribe an interim optimal price. Therefore, $p_{1}(c)=p_{2}(c)$ for almost all $c \in[0, \bar{c}]$, and in particular, every convex combination $p_{\alpha}(\cdot) \equiv \alpha p_{1}(\cdot)+(1-\alpha) p_{2}(\cdot)$ will be equivalent on $c \in[0, \bar{c}]$. For $c \in(\bar{c}, 1]$, note that if prices and ultimate trading probabilities are monotone, it must be that, for all such $c, q^{S}\left(p_{1}(c)\right)=0$ and $q^{S}\left(p_{2}(c)\right)=0$, and so $q^{S}\left(p_{\alpha}(c)\right)=0$ for all $\alpha \in[0,1]$ and for all $c \in(\bar{c}, 1]$. Therefore, we have $\Pi\left(p_{\alpha}(\cdot) \mid \omega\right)=\Pi\left(p_{1}(\cdot) \mid \omega\right)$, i.e., $p_{\alpha}(\cdot) \in K^{p}[\omega]$ for all $\alpha$, as claimed $Q E D$.

The next lemma states that reservation prices are continuous in $\omega$. With $r(v \mid \omega)=v-$

\footnotetext{
${ }^{25} f(x-0)$ is defined as the left hand $\operatorname{limit}, \lim _{\varepsilon>0, \varepsilon \rightarrow 0} f(x-\varepsilon) . f(x+0)$ is defined analogously.
} 
$(1-\delta) V^{B}(v \mid \omega)$, we need to show continuity of the value function $V^{B}(\cdot \mid \omega)$. We cannot directly apply Berge's Maximum theorem since payoffs $U^{B}(r, v \mid \omega)$ do not need to be continuous in $\omega$, because, for given $r$, the mass of sellers who offer $p \leq r, M^{S}(c(r \mid \omega) \mid \omega)$, can have a discontinuity. Therefore, we use the following trick: instead of choosing a reservation price $r$, buyers are thought of as choosing a threshold seller $c_{x}$ and trade with all sellers with $c \leq c_{x}$ :

Lemma $10 K^{r}[\cdot]$ is continuous in $\omega$.

Proof: Given $c_{x} \in[0,1]$, let the ultimate trading probability be $q_{x}^{B}\left(c_{x} \mid \omega\right)$, with $q_{x}^{B}\left(c_{x} \mid \omega\right) \equiv$ $\frac{M^{S}\left(c_{x}\right) M^{S}(1)^{-1}}{1-(1-\delta)\left(1-M^{S}\left(c_{x}\right) M^{S}(1)^{-1}\right)}$, and let expected prices be $E_{x}\left[p \mid c_{x}, \omega\right] \equiv \frac{1}{M^{S}\left(c_{x}\right)} \int_{0}^{c_{x}} p(c) d M^{S}(c)$. Then payoffs from trading with all $c \leq c_{x}$ are

$$
U_{x}^{B}\left(c_{x}, v \mid \omega\right) \equiv q_{x}^{B}\left(c_{x} \mid \omega\right)\left(v-E_{x}\left[p \mid c_{x}, \omega\right]\right)
$$

and clearly $U_{x}^{B}\left(c_{x}, v \mid \omega\right)$ is continuous in $c_{x}, v$, and $\omega$. Therefore $V_{x}^{B}(v \mid \omega)=\max _{c_{x}} U_{x}^{B}\left(c_{x}, v \mid \omega\right)$ is continuous in $\omega$ by the Maximum theorem. In addition, payoffs from maximizing with respect to cutoff types $c_{x}$ are equal to payoffs from maximizing with respect to cutoff prices $r$, i.e., $V_{x}^{B}(v \mid \omega)=V^{B}(v \mid \omega)$ : Whenever $p^{S}(\cdot)$ is increasing at $c_{x}$, this follows immediately by setting $r(v)=p\left(c_{x}\right)$; if $p^{S}(\cdot)$ is flat at the optimal cutoff $c_{x}$, the buyer must be indifferent between accepting and rejecting $p^{S}\left(c_{x}\right)$. Thus, the continuity of $V_{x}^{B}(\cdot \mid \cdot)$ carries over to $V^{B}(\cdot \mid \cdot) \quad Q E D$.

Now we want to show that the pool responses $K^{S}$ and $K^{B}$ are continuous. This is the main technical challenge of the existence proof. The problem here is that we need to evaluate composite functions. In particular, to calculate the trading probability of a type $v$, we need to evaluate the share $M^{S}(c(r(v)) \mid \omega) M^{S}(1 \mid \omega)^{-1}$. However, the type $c$ who trades with $v, c(r(v) \mid \omega)$, does not need to be continuous in $\omega$. Therefore, we need to state first three auxiliary lemmas to deal with the problem of composite (inverse) functions. The first lemma states a partial converse to Lebesgue's bounded convergence theorem:

Lemma 11 Let $\left\{f_{N}\right\}$ be a sequence of measurable functions $f_{N}:[0,1] \rightarrow[0,1]$ such that $f_{N} \rightarrow \bar{f}$ in $L_{1}$. Then $f_{N}(x) \rightarrow \bar{f}(x)$ pointwise for almost all $x \in[0,1]$ if a) all $f_{N}$ and $\bar{f}$ are weakly increasing or if b) the family $\left\{f_{N}\right\}$ is equicontinuous.

Proof: For the first part: We show convergence at all interior continuity points of $\bar{f}$ which implies the statement. Let $x^{\prime}$ be such a point, and suppose there is some 
subsequence such that $\lim f_{N^{\prime}}\left(x^{\prime}\right) \equiv f_{H} \neq \bar{f}\left(x^{\prime}\right) \equiv f_{x^{\prime}}$. Suppose $f_{H}>f_{x^{\prime}}$ (the other case is symmetric). Choose $\varepsilon$ such that for all $x \in B_{\varepsilon}\left(x^{\prime}\right),\left|\bar{f}\left(x^{\prime}\right)-\bar{f}(x)\right| \leq \frac{1}{2}\left|f_{H}-f_{x^{\prime}}\right|$, and choose any $x_{H} \in B_{\varepsilon}\left(x^{\prime}\right)$ such that $x_{H}>x^{\prime}$. By the monotonicity of each element $f_{N^{\prime}}$, $f_{N^{\prime}}(x) \geq f_{N^{\prime}}\left(x^{\prime}\right)$ for all $x \in\left[x^{\prime}, x_{H}\right]$ and for all $N^{\prime}$. Thus, $\liminf f_{N^{\prime}}(x) \geq f_{H}$. Hence, $\liminf \int_{x^{\prime}}^{x_{H}}\left|f_{N^{\prime}}(x)-\bar{f}(x)\right| d x \geq \frac{1}{2}\left(x_{H}-x^{\prime}\right)\left|f_{H}-f_{x^{\prime}}\right|>0$, contradicting $f_{N} \rightarrow \bar{f}$. The second part is immediate $Q E D$.

Take a sequence $\omega_{N}=\left[p_{N}, r_{N}, M_{N}^{S}, M_{N}^{B}\right]$ with $\omega_{N} \rightarrow \bar{\omega}$ : we want to show convergence of the composite functions $M_{N}^{B}\left(v_{N}\left(p_{N}(\cdot) \mid \omega_{N}\right)\right)$ and $M_{N}^{S}\left(c_{N}\left(r_{N}(\cdot) \mid \omega_{N}\right)\right)$. The former composite function is not a problem since the families $\left\{M_{N}^{B}(\cdot)\right\}$ and $\left\{v_{N}(\cdot)\right\}$ are equicontinuous and strictly increasing. Therefore, one can show that $M_{N}^{B}\left(v_{N}\left(p_{N}(c) \mid \omega_{N}\right)\right)$ converges pointwise. Here, we concentrate on $M_{N}^{S}\left(c_{N}\left(r_{N}(\cdot) \mid \omega_{N}\right)\right)$. The next lemma states that $c_{N}\left(r_{N}(v) \mid \omega_{N}\right)$ converges pointwise almost everywhere, which is a sufficient condition for the pointwise convergence of $M_{N}^{S}\left(c_{N}\left(r_{N}(v) \mid \omega_{N}\right)\right)$ :

Lemma 12 Given a sequence $\left\{p_{N}(\cdot)\right\}_{N=1}^{\infty}$ with $p_{N} \in \Sigma_{p(\cdot)}$ and given a sequence $\left\{r_{N}(\cdot)\right\}_{N=1}^{\infty}$ with $r_{N}(\cdot) \in \Sigma_{r(\cdot)}$, suppose $p_{N}(c) \rightarrow \bar{p}(c)$ and $r_{N}(v) \rightarrow \bar{r}(v)$ pointwise for almost every $c$ and $v$. Let $c\left(p \mid p_{N}(\cdot)\right) \equiv \sup \left\{c, 0 \mid p_{N}(c) \geq p\right\}$ and let $c_{N}(p) \equiv c\left(p \mid p_{N}(\cdot)\right)$. Then $c_{N}(p) \rightarrow \bar{c}(p) \equiv c(p \mid \bar{p}(\cdot))$ a.e. and $c_{N}\left(r_{N}(v)\right) \rightarrow \bar{c}(\bar{r}(v))$ a.e.

Proof: First, we show convergence of the inverse $c(\cdot \mid p(\cdot))$, starting by showing that we can disregard points where $p(\cdot)$ is flat because then the inverse function will have a jump, and these points have zero measure. At all $c$ where $p(\cdot)$ is not flat, convergence of the inverse function at $p(c)$ is not a problem. Then we prove convergence of the composite, making use of its monotonicity and continuity almost everywhere.

Suppose $\bar{p}(\cdot)$ is flat at $c_{f}$, i.e., for some $c_{f f} \neq c_{f}, \bar{p}\left(c_{f}\right)=\bar{p}\left(c_{f f}\right) \equiv p_{f}$ and suppose $p_{f} \in(0,1)$ and $c_{f}<c_{f f}$ (wlog). Then $c\left(p_{f}-0 \mid \bar{p}(\cdot)\right)<c\left(p_{f}+0 \mid \bar{p}(\cdot)\right)$ because $c\left(p_{f}-\varepsilon\right)=\sup \left\{c, 0 \mid \bar{p}(c) \leq p_{f}-\varepsilon\right\} \leq c_{f}$ and $c\left(p_{f}+\varepsilon\right)=\sup \left\{c, 0 \mid \bar{p}(c) \leq p_{f}+\varepsilon\right\} \geq$ $c_{f f}$. So $p_{f}$ is a jump point of $\bar{c}(\cdot)=c(\cdot \mid \bar{p}(\cdot))$. Let $p_{l} \equiv \bar{p}(0)$ and $p_{h} \equiv \bar{p}(1)$, then for all $p^{+}>p_{h}$ and $\varepsilon>0$, we have $\bar{p}(1-\varepsilon)<p^{+}$. Thus, at all $c^{\prime} \in(1-\varepsilon, 1)$ such that $p_{N}\left(c^{\prime}\right)$ converges, $\bar{p}\left(c^{\prime}\right)<p^{+}$, and for $N$ large enough, $p_{N}\left(c^{\prime}\right)<p^{+}$. Therefore $c_{N}\left(p^{+}\right) \geq c^{\prime}>1-\varepsilon$, and with $\varepsilon$ arbitrary, this implies $c_{N}\left(p^{+}\right) \rightarrow 1$ for all $p^{+}>p_{h}$. Reasoning similarly for $p^{-}<p_{l}$, we conclude that $c\left(\cdot \mid p_{N}(\cdot)\right)$ converges on $\left[0, p_{l}\right)$ and $\left(p_{h}, 1\right]$. Now, take any $p^{\prime} \in\left(p_{l}, p_{h}\right)$ such that $p^{\prime}$ is not a jump point of $\bar{c}(\cdot)$. Let $c^{\prime}=\bar{c}\left(p^{\prime}\right)$ and suppose $c^{\prime} \in(0,1)$. Then $\bar{p}(\cdot)$ is not flat at $c^{\prime}$, i.e., for all $c^{-}<c^{\prime}<c^{+}$, $\bar{p}\left(c^{-}\right)<\bar{p}\left(c^{\prime}\right)<\bar{p}\left(c^{+}\right)$. Take some $c^{-}$and $c^{+}$such that $p_{N}(\cdot)$ converges pointwise at 
these points. Then for some $\varepsilon$ and $N$ large enough, $p_{N}\left(c^{-}\right) \leq p^{\prime}-\varepsilon$ and $p_{N}\left(c^{+}\right) \geq p^{\prime}+\varepsilon$, and so $c_{N}\left(p^{\prime}\right)=\sup \left\{c, 0 \mid p_{N}(c) \leq p^{\prime}\right\} \in\left[c^{-}, c^{+}\right]$, and by $c^{-}$and $c^{+}$being arbitrary, $c_{N}\left(p^{\prime}\right) \rightarrow c^{\prime}$. Suppose $c^{\prime}=0$, then $\bar{p}(\varepsilon)>p^{\prime}$ for all $\varepsilon>0$ since $\bar{p}$ is not flat. Choose some $\varepsilon_{1}$ such that $p_{N}\left(\varepsilon_{1}\right) \rightarrow \bar{p}\left(\varepsilon_{1}\right)$. Then for $N$ large enough, $c_{N}\left(p^{\prime}\right) \leq \varepsilon_{1}$, and by $\varepsilon_{1}$ being chosen arbitrary, $c_{N}\left(p^{\prime}\right) \rightarrow 0$. Similar reasoning holds for $c^{\prime}=1$. Together, $c_{N}(p) \rightarrow \bar{c}(p)$ for almost all $p$.

Now we want to show $c_{N}\left(r_{N}(\cdot)\right) \rightarrow \bar{c}(\bar{r}(\cdot))$. Since $r(\cdot)$ is strictly increasing, we can disregard the zero measure set of $v$ where $\bar{c}(\cdot)$ is discontinuous at $\bar{r}(v)$. We also disregard the two points $v \in\{0,1\}$, noting that $\bar{r}(v) \in(0,1)$ at all $v \in(0,1)$. Note that at all remaining $v^{\prime}, \bar{c}(\cdot)$ will be continuous at $\bar{r}\left(v^{\prime}\right)$, and therefore $c_{N}\left(\bar{r}\left(v^{\prime}\right)\right)$ converges pointwise to $\bar{c}\left(\bar{r}\left(v^{\prime}\right)\right)$ by the reasoning in the preceding paragraph. Take such a type $v^{\prime} \in(0,1)$ and any $\delta_{1}<\min \left\{\bar{r}\left(v^{\prime}\right), 1-\bar{r}\left(v^{\prime}\right)\right\}$. Then we want to show that for every such $\delta_{1}$ there is some $N$ large enough such that $c_{N}\left(r_{N}\left(v^{\prime}\right)\right)$ is in an open ball with radius $\delta_{1}$ around $c^{\prime} \equiv \bar{c}\left(\bar{r}\left(v^{\prime}\right)\right)$, i.e., $c_{N}\left(r_{N}\left(v^{\prime}\right)\right)$ converges to $\bar{c}\left(\bar{r}\left(v^{\prime}\right)\right)$ pointwise: By continuity of $\bar{c}(\cdot)$ at $\bar{r}\left(v^{\prime}\right)$, there are some prices $p_{l}, p_{h}$ around $\bar{r}\left(v^{\prime}\right)$ with $p_{l}<\bar{r}\left(v^{\prime}\right)<p_{h}$ such that $\bar{c}\left(p_{l}\right) \in B_{\delta_{1}}\left(c^{\prime}\right) \equiv\left(c^{\prime}-\delta_{1}, c^{\prime}+\delta_{1}\right)$ and $\bar{c}\left(p_{h}\right) \in B_{\delta_{1}}\left(c^{\prime}\right)$. In addition, we choose these prices such that $c_{N}(\cdot)$ converges pointwise at $p_{l}$ and $p_{h}$. Such prices exist within the open ball because $c_{N}(\cdot)$ converges pointwise almost everywhere. By this choice there is some $N_{1}$ large enough such that $c_{N}\left(p_{l}\right) \in B_{\delta_{1}}\left(c^{\prime}\right)$ and $c_{N}\left(p_{h}\right) \in B_{\delta_{1}}\left(c^{\prime}\right)$ for $N \geq N_{1}$. Now choose $N_{2} \geq N_{1}$ such that $r_{N}\left(v^{\prime}\right) \in\left(p_{l}, p_{h}\right)$ for all $N \geq N_{2}$ as well. The monotonicity of $c_{N}(\cdot)$ implies that we have successfully sandwiched $c_{N}\left(r_{N}\left(v^{\prime}\right)\right)$, $c_{N}\left(p_{l}\right) \leq c_{N}\left(r_{N}\left(v^{\prime}\right)\right) \leq c_{N}\left(p_{h}\right)$, and from $c_{N}\left(p_{l}\right) \in B_{\delta_{1}}\left(c^{\prime}\right)$ and $c_{N}\left(p_{h}\right) \in B_{\delta_{1}}\left(c^{\prime}\right)$ we have $c_{N}\left(r_{N}\left(v^{\prime}\right)\right) \in B_{\delta_{1}}\left(c^{\prime}\right)$ with $c^{\prime}=\bar{c}\left(\bar{r}\left(v^{\prime}\right)\right)$ for all $N \geq N_{2}$. Since $\delta_{1}$ is arbitrary, it must be that $c_{N}\left(r_{N}\left(v^{\prime}\right)\right) \rightarrow \bar{c}\left(\bar{r}\left(v^{\prime}\right)\right)$ QED.

Pool response operators $K^{S}$ and $K^{B}$ are integrals over functions of $\omega$, and integrals are taken with respect to measures induced by $\omega$. To prove the continuity of this operator, we need the following technical lemma, which combines the idea of Lebesgue's convergence result for a sequence of functions with Helly's convergence result for a sequence of measures:

Lemma 13 Let $F_{N}$ be a sequence of c.d.f.s converging to some c.d.f. F almost everywhere and let $g_{N}:[0,1] \rightarrow[0,1]$ be a sequence of measurable functions converging almost everywhere to some function $g(\cdot)$. Then $\int_{0}^{1} g_{N} d F_{N} \rightarrow \int_{0}^{1} g d F$.

Proof: Note that $\int_{0}^{1} g_{N} d F_{N}-\int_{0}^{1} g d F=\int_{0}^{1}\left(g_{N}-g\right) d F_{N}+\int_{0}^{1} g d F_{N}-\int_{0}^{1} g d F$. The second term converges to zero by Helly's convergence theorem (Kolmogorov, Fomin 
p.370). For the first term note that by Egorov's theorem (Kolmogorov, Fomin, p.290), for every $\varepsilon>0, g_{N}$ converges to $g$ uniformly on a measurable subset $A \subset[0,1]$ such that the remaining set is of measure $\varepsilon / 2$, i.e., $\varepsilon / 2 \geq \int_{[0,1]-A} d F(x)$. By Helly's convergence theorem, $\int_{x \in[0,1]-A} d F_{N}(x) \rightarrow \int_{x \in[0,1]-A} d F(x)$, and thus for some $N^{\prime}$, $\varepsilon \geq \int_{x \in[0,1]-A} d F_{N}(x) \geq \int_{x \in[0,1]-A}\left|g_{N}(x)-g(x)\right| d F_{N}(x)$ for all $N \geq N^{\prime}$. By $g_{N} \rightarrow g$ uniformly on $A$, there is some $N^{\prime \prime}$ such that $\left|g_{N}(x)-g(x)\right| \leq \varepsilon$ for all $x \in A$ and $N \geq N^{\prime \prime}$. Thus, for all $N \geq \max \left\{N^{\prime}, N^{\prime \prime}\right\}, \int_{0}^{1}\left(g_{N}-g\right) d F_{N}=\int_{A}\left(g_{N}-g\right) d F_{N}+$ $\int_{[0,1]-A}\left(g_{N}-g\right) d F_{N} \leq 2 \varepsilon$ and since $\varepsilon$ is arbitrary, the claim follows $Q E D$.

From the latter two lemmas, we get

Lemma $14 K^{S}$ and $K^{B}$ are continuous.

Proof: From Lemma 12, $D(p(c) \mid \cdot) \equiv M^{B}(v(p(c) \mid \cdot)) M^{B}(1)$ and $S(r(v) \mid \cdot) \equiv M^{S}(c(r(v) \mid \cdot)) M^{S}(1)$ are pointwise continuous in $\omega$. From Lemma 13, this carries over to $K^{S}[\cdot]$ and $K^{B}[\cdot] \quad Q E D$.

A final auxiliary lemma states that if the steady-state conditions hold, then the mass of sellers and the mass of buyers must be identical:

Lemma 15 If $\omega$ is a fixed point of $K$, then $M^{S}(1)=M^{B}(0)$.

Proof: Let $x^{S}$ and $x^{B}$ be the shares of sellers and buyers, respectively, who trade:

$$
\begin{aligned}
& x^{S}=M^{S}(1)^{-1} \int_{0}^{1}\left[\int_{v(p(c))}^{1}\left[d M^{B}(v)\right] M^{B}(0)^{-1}\right] d M^{S}(c) \\
& x^{B}=M^{B}(0)^{-1} \int_{0}^{1}\left[\int_{0}^{c(r(v))} d M^{S}(c) M^{S}(1)^{-1}\right] d M^{B}(v),
\end{aligned}
$$

and note that by the same reasoning as for Lemma 2, the share of sellers and buyers who trade must be the same:

$$
\begin{aligned}
x^{S} & =M^{S}(1)^{-1} \int_{0}^{1}\left[\int_{v(p(c))}^{1}\left[d M^{B}(v)\right] M^{B}(0)^{-1}\right] d M^{S}(c) \\
& =\int_{0}^{1}\left[\int_{v(p(c))}^{1}\left(\left[d M^{B}(v)\right] M^{B}(0)^{-1}\right)\right]\left(d M^{S}(c) M^{S}(1)^{-1}\right) \\
& =\int_{0}^{1}\left[\int_{0}^{c(r(v))}\left(d M^{S}(c) M^{S}(1)^{-1}\right)\right]\left(\left[d M^{B}(v)\right] M^{B}(0)^{-1}\right) \\
& =x^{B},
\end{aligned}
$$


and by rewriting the steady-state conditions we get

$$
\begin{aligned}
G^{S}(1) & =x^{S} M^{S}(1)+M^{S}(1)\left(1-x^{S}\right) \delta \\
G^{B}(1) & =x^{B} M^{B}(0)+M^{B}(0)\left(1-x^{B}\right) \delta,
\end{aligned}
$$

so that $x^{S}=x^{B}$ implies $M^{S}(1)=M^{B}(0)$ by rewriting further:

$$
\begin{aligned}
& 1=M^{S}(1)\left(x^{S}+\left(1-x^{S}\right) \delta\right) \\
& 1=M^{B}(0)\left(x^{B}+\left(1-x^{B}\right) \delta\right)
\end{aligned}
$$

Theorem 3 (Restated): For every $\delta$ there exists an equilibrium $\sigma^{*}$.

Proof: Bounded monotone functions are compact in the locally convex Hausdorff space $L_{1}$ (Helly's selection principle implies that this set is sequentially compact and sequential compactness is sufficient for compactness in metric spaces). Monotone functions with a bounded slope as defined here form a closed subset of the monotone functions, and hence, these sets are compact. Therefore, the set $\Omega \subset L_{1}$, endowed with the integral norm, is compact. Convexity of $\Omega$ is immediate. Together with the above Lemmas, the correspondence $K$ satisfies the conditions of the Kakutani-Fan-Glicksberg fixed point theorem. Thus, there exists some $\omega^{*} \in \Omega$ such that $\omega^{*} \in K\left[\omega^{*}\right]$.

The fixed point $\omega^{*}=\left[p^{*}, r^{*}, M^{S *}, M^{B *}\right]$ corresponds to a steady-state equilibrium $\sigma^{*}$, and we show how to translate it. First, we need to translate the masses $M^{S}$ and $M^{B}$ into shares $\Phi^{S}$ and $\Phi^{B}$. For this, let $M^{*} \equiv M^{S *}(1), \Phi^{S *}(c) \equiv M^{S}(c) M^{*-1}$, and $\Phi^{B *}(v) \equiv\left(M^{*}-M^{B}(v)\right) M^{*-1} \cdot{ }^{26}$ The identity $M^{S *}(1)=M^{B *}(1)$ follows from Lemma (15). Now, we translate the ex ante optimal pricing function $p^{*}$ into an interim optimal pricing function $\bar{p}$ : Let $\bar{p}(\cdot)$ be equal to $p^{*}(\cdot)$ whenever $p^{*}(c)$ is interim optimal. For all other points $c \in[0,1)$, take the right limit, $\bar{p}(c)=\lim _{\varepsilon \rightarrow 0, \varepsilon>0} p^{*}(c+\varepsilon)$, which preserves monotonicity and interim optimality by continuity of $U^{S}(\cdot, \cdot)$. Finally, let $\bar{p}(1)=\lim _{\varepsilon \rightarrow 0, \varepsilon>0} p^{*}(1-\varepsilon)$. Recall that the set of types for which $p^{*}(\cdot)$ is not interim optimal has measure zero. Changing prices on this set does therefore not change the distribution of price offers so that neither steady-state conditions nor buyers' optimality conditions are affected. Hence, $\sigma^{*} \equiv\left[\bar{p}(\cdot), r^{*}(\cdot), \Phi^{S *}(\cdot), \Phi^{B *}(\cdot), M^{*}\right]$ is a steady-state equilibrium $Q E D$.

\footnotetext{
${ }^{26}$ Note that $\Phi^{B}(v)$ is the share of types below $v$, while $M^{B}(v)$ is the mass of types above $v$.
} 
Remark 1 Lemma 14 together with the reasoning in the proof of the last theorem, implies Theorem 2: For fixed $p(\cdot)$ and $r(\cdot)$, the joint pool response $\left(K^{S}, K^{B}\right)[p(\cdot), r(\cdot), \cdot, \cdot]$ defines a function which maps the compact subset $\Sigma_{M^{S}} \times \Sigma_{M^{B}} \subset L_{1}$ into itself. In particular, for fixed $p(\cdot)$ and $r(\cdot)$, the pool response $\left(K^{S}, K^{B}\right)[p(\cdot), r(\cdot), \cdot, \cdot]$ is continuous in $M^{S}$ and $M^{B}$. Thus, according to the Kakutani-Fan-Glicksberg theorem, there exists a fixed point, i.e., some $\left(\bar{M}^{B}, \bar{M}^{S}\right)$ such that $\left(K^{S}, K^{B}\right)\left[p(\cdot), r(\cdot), \bar{M}^{B}, \bar{M}^{S}\right]=$ $\left(\bar{M}^{B}, \bar{M}^{S}\right)$. As shown in the preceding proof, if $K^{S}\left[\bar{M}^{B}, \bar{M}^{S}\right]=\bar{M}^{S}$ and similarly $K^{B}\left[p(\cdot), r(\cdot), \bar{M}^{B}, \bar{M}^{S}\right]=\bar{M}^{B}$, then $\left(\bar{M}, \bar{\Phi}^{S}, \bar{\Phi}^{B}\right)$ - defined by $\bar{M} \equiv \bar{M}^{S}(1), \bar{\Phi}^{S}(c) \equiv$ $\bar{M}^{S}(c) \bar{M}^{-1}$, and $\bar{\Phi}^{B}(v) \equiv\left(\bar{M}-\bar{M}^{B}(v)\right) \bar{M}^{-1}$ - satisfy the steady-state conditions (6) and (7) for given $p(\cdot)$ and $r(\cdot)$.

\section{B.5 Proof of Lemmas 3, 4, 5, and 7 .}

Proof of Lemma 3: From $r_{k^{\prime}}(1)=1-\left(1-\delta_{k^{\prime}}\right) V\left(1 \mid \sigma_{k^{\prime}}^{*}, \delta_{k^{\prime}}\right)$, and from $V\left(1 \mid \sigma_{k^{\prime}}^{*}, \delta_{k^{\prime}}\right) \geq$ $U^{B}\left(r_{k^{\prime}}(v), 1 \mid \sigma_{k^{\prime}}^{*}, \delta_{k^{\prime}}\right)$, with $U^{B}\left(r_{k^{\prime}}(v), 1 \mid \sigma_{k^{\prime}}^{*}, \delta_{k^{\prime}}\right)=Q_{k^{\prime}}^{B}(v)\left(1-E\left[p \mid p \leq r_{k^{\prime}}(v)\right]\right)$, we get

$$
\liminf r_{k^{\prime}}(1)<1-(1-h) \limsup Q_{k^{\prime}}^{B}(v) \quad \forall v<h,
$$

observing that $r_{k^{\prime}}(v) \leq v$ implies that $E\left[p \mid p \leq r_{k^{\prime}}(v)\right]<h$ for all $v<h$. The lemma follows since $\lim \sup Q_{k^{\prime}}^{B}(v)=1$ would imply $\lim \inf r_{k^{\prime}}(1)<h$, contradicting the definition of $h \quad Q E D$

Proof of Lemma 4: From $p_{k^{\prime}}(c) \geq l_{k^{\prime}}$ for all $c$, it follows that $E[p \mid p \leq r] \geq l_{k^{\prime}}$ for all $r \geq l_{k^{\prime}}$ and $q_{k^{\prime}}^{B}\left(r \mid \sigma_{k^{\prime}}^{*}, \delta_{k^{\prime}}\right)=0$ for all $r<l_{k^{\prime}}$. Therefore, $U^{B}\left(r_{k^{\prime}}(v), v \mid \sigma_{k^{\prime}}^{*}, \delta_{k^{\prime}}\right) \leq$ $Q_{k^{\prime}}^{B}(v)(v-l)$ for all $v$. With $\lim \sup Q_{k^{\prime}}^{B}(v)<1$ from Lemma 3, this implies $\lim \sup V\left(v \mid \sigma_{k^{\prime}}^{*}, \delta_{k^{\prime}}\right)<v-l$ for $v \in(l, h)$, and hence, $\liminf r_{k^{\prime}}(v)=v-\lim \sup V\left(v \mid \sigma_{k^{\prime}}^{*}, \delta_{k^{\prime}}\right)>$ $l \quad Q E D$.

Proof of Lemma 5: Manipulation of the steady-state condition shows that the share of types is proportional to their probability of not trading: ${ }^{27}$

$$
1-\Phi_{k^{\prime}}^{B}(v)=\int_{v}^{1} \frac{1-Q_{k^{\prime}}^{B}(\tau)+\delta Q_{k^{\prime}}^{B}(\tau)}{M_{k^{\prime}} \delta_{k^{\prime}}} d G^{B}(\tau) .
$$

\footnotetext{
${ }^{27}$ Rewriting the definition of $q^{B}$, one gets $\frac{1}{(1-(1-\delta)(1-S(r(v))))}=\frac{q^{B}(r(v))\left(1-q^{B}(r(v))+\delta q^{B}(r(v))\right)}{\delta q^{B}(r(v))}$, and rewriting (7) shows that $1-\Phi^{B}(v)=\int_{0}^{v}(1-(1-\delta)(1-S(r(v))))^{-1} M^{-1} d G^{B}(v)$.
} 
The total mass $M_{k^{\prime}}$ is at most $\frac{1}{\delta_{k^{\prime}}}$, as can be seen by evaluating the steady-state condition (7) at $v=1$. Therefore, $M_{k^{\prime}} \delta_{k^{\prime}}$ must be smaller than 1 . We use this to rewrite the last equation to get a lower bound

$$
1-\Phi_{k^{\prime}}^{B}(v) \geq \int_{v}^{v+\varepsilon}\left(1-Q_{k^{\prime}}^{B}(\tau)\right) d G^{B}(\tau)
$$

where $\varepsilon \geq 0$. Let $(1-\bar{Q}) \equiv \liminf \left(1-Q_{k^{\prime}}^{B}(v+\varepsilon)\right)$. If $\varepsilon \in(0, h-v)$, then from the last lemma, we know that $(1-\bar{Q})>0$, and from the monotonicity of $r_{k^{\prime}}(\cdot)$, we have $\left(1-Q_{k^{\prime}}^{B}(\tau)\right) \geq(1-\bar{Q})$ for all $\tau \leq v+\varepsilon$, and thus,

$$
\liminf 1-\Phi_{k^{\prime}}^{B}(v) \geq(1-\bar{Q})\left(G^{B}(v+\varepsilon)-G^{B}(v)\right)>0
$$

where we use the assumption that static demand $\left(1-G^{B}(\cdot)\right)$ is strictly decreasing, which implies that $\left(G^{B}(v+\varepsilon)-G^{B}(v)\right)>0 \quad Q E D$.

Proof of Lemma 7: From $\liminf p_{k^{\prime}}(c) \geq p^{c}$ for all $c$ and $r_{k^{\prime}}(v) \leq v, \lim Q_{k^{\prime}}^{B}(v)=0$ for all $v<p^{c}$. For $v>p^{c}$, limiting payoffs are bounded from above by $\max \left\{v-p^{c}, 0\right\}$ and thus, $\liminf r_{k^{\prime}}(v) \geq p^{c}$. From the definition of $h_{k^{\prime}}$ and by monotonicity of $r_{k^{\prime}}$, $r_{k^{\prime}}(v) \leq h_{k^{\prime}}$ for all $v$. Together, $\lim r_{k^{\prime}}(v)=\lim h_{k^{\prime}}=p^{c}$ for all $v>p^{c}$. The expected price conditional on trading becomes $p^{c}, E\left[p \mid p \leq r_{k^{\prime}}(v)\right] \rightarrow p^{c}$ for all $v>p^{c}$. Rewriting the equilibrium condition for $r_{k^{\prime}}$ shows:

$$
\lim _{k^{\prime} \rightarrow \infty} r_{k^{\prime}}(v)=v-\lim _{k^{\prime} \rightarrow \infty} Q_{k^{\prime}}^{B}(v)\left(v-p^{c}\right)
$$

from which $\lim r_{k^{\prime}}(v)=p^{c}$ only if $\lim Q_{k^{\prime}}^{B}(v)=1 \quad Q E D$. 


\section{References}

[1] Aliprantis, Charalambos; Border, Kim (1994): "Infinite Dimensional Analysis," Berlin; Springer.

[2] Atakan, Alp (2007): "Competitive Equilibria in Decentralized Matching with Incomplete Information," Mimeo, Northwestern University.

[3] Burdett, Kenneth; Judd, Kenneth (1983): "Equilibrium Price Dispersion," Econometrica, Vol. 51, 955-969.

[4] Butters, Gerard R. (1977): "Equilibrium Price Distributions in a Random Meetings Market," unpublished manuscript, Princeton.

[5] Butters, Gerard R. (1977): "Equilibrium Distributions of Sales and Advertising Prices," The Review of Economic Studies, Vol. 44 , 465-491.

[6] De Fraja, Gianni; Sakovics, Jozsef (2001): "Walras Retrouve: Decentralized Trading Mechanisms and the Competitive Price," Journal of Political Economy, Vol 109, 842-863.

[7] Gale, Douglas (1987): "Limit Theorems for Markets with Sequential Bargaining," Journal of Economic Theory, Vol 43, 20-54.

[8] Gale, Douglas (2000): "Strategic Foundations of General Equilibrium: Dynamic Matching and Bargaining Games," Churchill Lectures in Economic Theory, Cambridge.

[9] Diamond, Peter A. (1971): "A Model of Price Adjustment," Journal of Economic Theory, 158-68.

[10] Hendel, Igal; Nevo, Aviv (2006): "Measuring the Implications of Sales and Consumer Inventory Behavior," Econometrica, Vol 74, 1637 - 1673.

[11] Inderst, Roman (2001): "Screening in a Matching Market," The Review of Economic Studies, Vol. 68, 849-68.

[12] Kolmogorov, Aleksey; Fomin, Sergei Vasilovich (1970): "Introductory Real Analysis," New York; Dover Publishing.

[13] Lauermann, Stephan (2006a): "When Less Information is Good for Efficiency: Private Information in Bilateral Trade and in Markets," Mimeo, University of Michigan. 
[14] Lauermann, Stephan (2006b): "Dynamic Matching and Bargaining Games: A General Approach," Mimeo, University of Michigan.

[15] McAfee, Preston R (1993): "Mechanism Design by Competing Sellers," Econometrica, Vol. 61, 1281-1312.

[16] McMillan, John; Rothschild, Michael (1994): "Search," in: Robert Aumann and Sergiu Hart (eds.), Handbook of Game Theory, Vol 2, Elsevier.

[17] Milgrom, Paul; Segal, Ilya (2002): "Envelope Theorems for Arbitrary Choice Sets," Econometrica, Vol. 70, 583-601.

[18] Milgrom, Paul; Shannon, Chris (1994): "Monotone Comparative Statics," Econometrica, Vol. 62, 157-180.

[19] Moreno, Diego; Wooders, John (2002): "Prices, Delays and Dynamics of Trade," Journal of Economic Theory, Vol 104, 304-339.

[20] Mortensen, Dale; Wright, Randy (2002): "Competitive Pricing and Efficiency in Search Equilibrium," International Economic Review, 43, 1-20.

[21] Mylovanov, Tymofiy; Tröger, Thomas (2007): "Informed Principal in Quasi-Linear Environments," Mimeo, University of Bonn.

[22] Rubinstein, Ariel; Wolinsky, Asher (1985): "Equilibrium in a Market with Sequential Bargaining," Econometrica, Vol 53, 1133-50.

[23] Samuelson, Larry (1992): Disagreement in Markets with Matching and Bargaining, The Review of Economic Studies, Vol 59, 177-185.

[24] Satterthwaite, Mark; Shneyerov, Artyom (2007a): "Dynamic Matching, Two-Sided Incomplete Information and Participation Costs: Existence and Convergence to Perfect Competition," Econometrica, Vol 75, 155-200.

[25] Satterthwaite, Mark; Shneyerov, Artyom (July, 2007b): "Convergence of a Dynamic Matching and Bargaining Market with Two-sided Incomplete Information to Perfect Competition," Mimeo, Northwestern University.

[26] Serrano, Roberto (2002): "Decentralized Information and the Walrasian Outcome: A Pairwise Meetings Markets with Private Information," Journal of Mathematical Economics, Vol 38, 65-89. 
[27] Shneyerov, Artyom; Wong, Adam (2007): "Bilateral Matching and Bargaining with Private Information," Mimeo, University of British Columbia.

[28] Yilankaya, Okan (1999): "A Note on the Seller's Optimal Mechanism in Bilateral Trade with Two-Sided Incomplete Information," Journal of Economic Theory, Vol 87, 125-143. 